\title{
SPATIAL EXTERNALITY AND INDETERMINACY
}

\author{
Emmanuelle Augeraud-VÉron ${ }^{1, *}$ And Arnaud Ducrot ${ }^{2}$
}

\begin{abstract}
We study conditions for existence and uniqueness of solutions in some space-structured economic models with long-distance interactions between locations. The solution of these models satisfies non local equations, in which the interactions are modeled by convolution terms. Using properties of the spectrum location obtained by studying the characteristic equation, we derive conditions for the existence and uniqueness of the solution. This enables us to characterize the degree of indeterminacy of the system being considered. We apply our methodology to a theoretical one-sector growth model with increasing returns, which takes into account technological interdependencies among countries that are modeled by spatial externalities. When symmetric interaction kernels are considered, we prove that conditions for which indeterminacy occurs are the same as the ones needed when no interactions are taken into account. For Gaussian kernels, we study the impact of the standard deviation parameter on the degree of indeterminacy. We prove that when some asymmetric kernels are considered, indeterminacy can occur with classical assumptions on supply and demand curves.
\end{abstract}

Mathematics Subject Classification. 91B72, 91B62, 49K20.

Received April 30, 2018. Accepted January 22, 2019.

\section{INTRODUCTION}

Spatial distribution is an important component of economic activity. This naturally appears through trade but also through spatial externalities: knowledge accumulated in a region is affected by knowledge accumulated in the surrounding areas. Klenow and Rodiguez-Clare [31] show that international knowledge spillovers are generated by both physical capital and human capital. Therefore, there are technological interdependencies between localities. These spatial externalities play a role in the process of economic growth. Many empirical studies have shown the importance of these spillovers on economic growth, both on a national level [21] and on a regional level (Rey and Montouri [38] for American States). According to Quah [36], "spatial spillover factors matter more than national, macro ones".

Spatial interactions may be introduced in two ways: either by considering capital or labor flow between regions or by considering long-distance interdependencies among regions. These interdependencies may correspond, for example, to technological interdependencies, modeled by capital or labor externalities.

The first case is studied for example in Quah [37], Camacho et al. [15], Boucekkine et al. [8], Brito [10, 11], Zhou [40]. This modeling reflects the mobility of capital or individuals, and implies a physical diffusion of

Keywords and phrases: Indeterminacy, spatial externalities.

1 Bordeaux University, GREThA (UMR CNRS 5113), Pessac, France.

2 Normandie Univ, UNIHAVRE, LMAH, FR-CNRS-3335, ISCN, Le Havre, France.

* Corresponding author: emmanuelle.augeraud@u-bordeaux.fr

(C) The authors. Published by EDP Sciences, 2019

This is an Open Access article distributed under the terms of the Creative Commons Attribution License (http://creativecommons.org/licenses/by/4.0), which permits unrestricted use, distribution, and reproduction in any medium, provided the original work is properly cited. 
human or physical capital. The dynamics of these spatial models is mainly given by diffusion and advection equations.

In the second case, spatial interdependencies involve long-distance interaction among agents, which results in the interaction of individuals in different places, without implying any motion. In this case, the dynamics is given by a non local system of equations. These non local interactions can be modeled using convolution kernels, which enable geographical distance between countries to be taken into account. Brock and Xepapadeas [13] emphasize that only considering the few first moments fails to capture the interaction between local centripetal forces and more distant centrifugal forces [24, 32]. In addition, considering general interaction kernels enables differential weights of neighborhood effects to be considered. These effects may be due to the distance between economic agents but also to asymmetric relationships between neighbors. Non local interaction kernels play a key part in explaining the role of conurbations [34].

One of the questions raised by studying spatial dynamics is the one of long run convergence to either a homogeneous distribution or to spatial patterns [13]. However, as our dynamics is built with predetermined and forward variables, the question of existence, uniqueness or indeterminacy of the solution should be considered beforehand.

The key part of our study is, given an initial condition for the predetermined variable, to find conditions under which the system of non local equations has a solution and whether or not this solution is unique. Since the seminal work of Blanchard and Kahn [6] and Buiter [14], this issue has been well known for finite dimensional systems like ODEs. Blanchard-Kahn conditions are obtained by comparing the dimension of the set of predetermined variables and the dimension of the stable manifold. If these two quantities are equal, there is existence and uniqueness of the solution of the local problem in the neighborhood of the steady state. This question has also been dealt with in an infinite dimensional setting, considering functional differential equations of mixed type (MFDEs), which arise in models with delay and advance. For these equations, Blanchard-Kahn conditions cannot be used, as the spaces being considered are infinite-dimensional. Mallet Paret and Verduyn Lunel have proved that for MFDEs, exponential dichotomies can be obtained. They factorize the characteristic function with two terms: one corresponding to the characteristic equation of a delay equation, the other to an advance equation. The roots of each of these two characteristic equations are isolated, and their location enables the calculation of an integer, which is an invariant of the system. This integer indicates the number of misplaced roots. As far as scalar systems are concerned, the value of this integer determines whether or not there is existence or uniqueness of the solution. In a series of works, Hupkes and Augeraud [29] and d'Albis et al. $[1,2]$ have stated a method based on regular perturbation of the system to compute this invariant for MFDEs or mixed functional algebraic equations (MFAEs).

For non local equations, such a study had not previously been carried out. As far as spatial interactions are concerned, Desmet and Rossi-Hansberg [19] underline the lack of mathematical theory to solve such questions. The aim of our study is to give an answer to this question.

As far as non local equations are concerned, the spectrum of the characteristic equation is a continuous set (when space is non periodic), obtained as a set which is parametrized by the Fourier modes, which appear following the Fourier Transform of the problem. This continuity of the spectrum is a situation that does not occur for ODEs (for which the number of characteristic roots is finite) or MFDEs (for which there is an infinite number of isolated characteristic roots). One of the main problems driven by this continuity is to deal with the spectrum which may cross the axis of roots with zero real part, as exponential dichotomies cannot be obtained in such a situation. Under some easy-to-check assumptions on this crossing, we prove a dichotomy property and determine conditions under which there is existence and uniqueness. Moreover, we develop mathematical tools that enable us to provide a simple criterion to characterize indetermination, and to compute the degree of indeterminacy for non local dynamics with forward-looking agents.

As far as indeterminacy in spatial models is concerned, one of the economic questions that is raised is whether or not spatial interactions between agents enlarge the set of parameters leading to indeterminacy. Another one is the role of interaction on the degree of indeterminacy.

To our knowledge, the first question has not been assessed for spatial models. However, a similar question has been studied for specific kinds of structured models, such as those for which the space dimension 
does not model geographical distance between agents but another type of heterogeneity, such as heterogeneity with respect to initial stock of capital and to labor endowments [28] or heterogeneity with respect to preferences [27].

The role of heterogeneity on indeterminacy is known to be ambiguous. According to Herrendorf et al. [28], indeterminacy is overstated in representative agent models, and a "stabilizing effect of heterogeneity" exists. Bosi and Seegmuller [7] also prove that heterogeneity may reduce the set of parameters compatible with indeterminacy. It has been proved in Ghiglino and Olszak-Duquenne [27] that the impact of heterogeneity on indeterminacy depends on the inverse of the absolute risk aversion function.

The article is organized as follows. In Section 2, we present a simple economic example in order to illustrate the equations we are going to study. We then consider a general version of the equation, with space variables being in $\mathbb{R}^{p}$ and present the questions raised by this equation. In Section 3, firstly we define the concept of forward solution for the algebraic-differential equation and define the notion of stable dichotomy. Our main result is given in Theorems 3.7 and 3.8. They establish the existence of a state space decomposition in which the corresponding linear operator generates a stable semigroup on the stable space and a backward stable semigroup on the unstable part. In Section 4, we study the issue of existence and uniqueness for the example set out in Section 1. We define the notion of degree of indeterminacy. We discuss the indeterminacy problem, according to the shape of the long distance interaction kernel.

\section{Presentation of the Problem}

\subsection{A simple example}

To motivate the equations we are going to study, we consider here a simple example which is derived from the growth model considered by Benhabib and Farmer [4].

In the case of Benhabib and Farmer [4] model's, there is no spatial dimension. We would call this case the benchmark model. This benchmark model is a classical closed economy where stand rational producers and consumers.

The production function of the representative firm is given by production function $f(k, l, A)=A(t) k(t)^{a} l(t)^{b}$, where $A$ is an externality, $k$ is capital and $l$ is labor. We assume that capital depreciate at rate $\delta$, and thus profit function of a representative price taker firm is given by $A(t) k(t)^{a} l(t)^{b}-r(t) k(t)-w(t) l(t)$, where $r(t)$ and $w(t)$ are respectively interest rate and wage rate. Maximization of profit yields usual conditions $r(t)=$ $a A(t) k(t)^{a-1} l(t)^{b}-\delta k(t)$ and $w(t)=b A(t) k(t)^{a} l(t)^{b-1}$.

The representative infinitely-lived individual maximizes intertemporal utility

$$
\int_{0}^{\infty} \tilde{u}(c, l) e^{-\rho t} \mathrm{~d} t
$$

under budgetary constraint

$$
\frac{\mathrm{d} a(t)}{\mathrm{d} t}=a(t) r(t)+w(t) l(t)-c(t)
$$

where $\rho$ is the intertemporal discount rate and while $c$ and $l$ respectively denote the consumption and the labour supply. When instantaneous utility function reads as

$$
\tilde{u}(c, l)=\ln (c)-\frac{l^{1-\chi}}{1-\chi},
$$


wherein $\chi \leq 0$, necessary and sufficient optimality conditions reads

$$
\left\{\begin{array}{l}
\frac{\mathrm{d} a(t)}{\mathrm{d} t}=a(t) r(t)+w(t) l(t)-\lambda(t)^{-1}, \\
\frac{\mathrm{d} \lambda(t)}{\mathrm{d} t}=-\lambda(t)[r(t)-\rho] \\
l(t)^{-\chi}=\lambda(t) w(t)
\end{array}\right.
$$

supplemented with transversality condition $\lim _{t \rightarrow \infty} \lambda(t) a(t) e^{-\rho t}=0$.

Under closed economy assumption, equilibrium is characterized by conditions $a(t)=k(t)$. Thus the dynamics can be described by a system of differential-algebraic equation with one predetermined variable and two forward variables, that reads as

$$
\left\{\begin{array}{l}
\frac{\mathrm{d} k(t)}{\mathrm{d} t}=A(t) k(t)^{a} l(t)^{b}-\delta k(t)-\lambda(t)^{-1} \\
\frac{\mathrm{d} \lambda(t)}{\mathrm{d} t}=-\lambda(t)\left[a A(t) k(t)^{a-1} l(t)^{b}-\delta-\rho\right] \\
l(t)^{-\chi}=\lambda(t) b A(t) k(t)^{a} l(t)^{b-1}
\end{array}\right.
$$

supplemented with the given initial condition $k(0)=k_{0} \in \mathbb{R}_{+}$and the transversality condition $\lim _{t \rightarrow \infty} \lambda(t) k(t) e^{-\rho t}=0$. We assume that the externalities take the form

$$
A(t)=\left(K^{e}(t)\right)^{\alpha-a}\left(L^{e}(t)\right)^{\beta-b}
$$

In the benchmark model, these externalities are fully characterized by $K^{e}(t)=k(t)$ and $L^{e}(t)=l(t)$. We assume that $1>\alpha>a>0, \beta>b>0$ with $\alpha+\beta>1$ and $a+b=1$.

We now consider a spatially distributed version of the above model, assuming that in each location $x \in \mathbb{R}^{p}$, agents are in a closed economy and that markets clear in each location $x \in \mathbb{R}^{p}$. In order to take into account spacial dependence, we would denote variables $V$ as $V(x, t)$. Let us also assume that $K^{e}(x, t)$ and $L^{e}(x, t)$ now capture technological interdependences among the spatial locations. To take into account the weight due to the geographical distance between regions, we consider that $K^{e}$ and $L^{e}$ are defined as convolution products and that they read as

$$
\begin{aligned}
K^{e}(x, t) & =\int_{\mathbb{R}^{p}} k(x-y, t) \phi_{1}(\mathrm{~d} y):=\phi_{1} * k(\cdot, t)(x), \\
L^{e}(x, t) & =\int_{\mathbb{R}^{p}} l(x-y, t) \phi_{2}(\mathrm{~d} y):=\phi_{2} * l(\cdot, t)(x),
\end{aligned}
$$

wherein $\phi_{1}$ and $\phi_{2}$ are two given non negative integrable functions on $\mathbb{R}^{p}$.

This modeling aims to take into account international diffusion of knowledge and ideas [22, 30]. The interactions between the regions only happen through these spatial externalities.

For this spatial economy with long-distance interactions, the model is derived as in the benchmark model. In each location $x \in \mathbb{R}$ representative price-taker firms maximize profits. In each location $x \in \mathbb{R}$ necessary and sufficient conditions are computed by the representative consumer, by maximizing intertemporal utility under budgetary condition. Thus, in each location $x \in \mathbb{R}$, the equilibrium solves the following non linear differentialalgebraic system of equations:

$$
\left\{\begin{array}{l}
\frac{\partial k(x, t)}{\partial t}=\left(\phi_{1} * k(x, t)\right)^{\alpha-a}\left(\phi_{2} * l(x, t)\right)^{\beta-b} k(x, t)^{a} l(x, t)^{b}-\delta k(x, t)-\lambda(x, t)^{-1} \\
\frac{\partial \lambda(x, t)}{\partial t}=-\lambda(x, t)\left[a\left(\phi_{1} * k(x, t)\right)^{\alpha-a}\left(\phi_{2} * l(x, t)\right)^{\beta-b} k(x, t)^{a-1} l(x, t)^{b}-\delta-\rho\right] \\
l^{-\chi}(x, t)=\lambda(x, t) b\left(\phi_{1} * k\right)^{\alpha-a}\left(\phi_{2} * l\right)^{\beta-b} k^{a} l^{b-1},
\end{array}\right.
$$


supplemented together with the transversality condition

$$
\lim _{t \rightarrow \infty} \lambda(x, t) k(x, t) e^{-\rho t}=0,
$$

and some suitable initial condition $k(x, 0)=k_{0}(x)$.

As each location acts as a closed economy, the derivation of the optimality condition in the spatial model are very similar to the one obtained in the benchmark one.

On the contrary to (2.1), $l$ can no more be explicitly expressed as a function of $k$ and $\lambda$. This is why the structure of algebraic equation prevails. We also notice that when $\phi_{1}$ and $\phi_{2}$ formally take the form of Dirac delta functions at zero, ${ }^{1}$ (which means that there are no interdependency among locations), then (2.2) can be re-written as System (2.1), for each $x \in \mathbb{R}^{p}$.

Observe now that System (2.2) admit a unique spatially homogeneous steady state $\left(k^{*}, l^{*}, \lambda^{*}\right)$, characterized by the following expression

$$
\left\{\begin{array}{l}
k^{*}=\left(\frac{\delta+\rho}{a}\right)^{\frac{(1-\beta-\chi)}{(1-\chi)(1-\alpha)}}\left(\frac{\delta(1-a)+\rho}{a b}\right)^{\frac{-\beta}{(1-\chi)(1-\alpha)}} \\
\lambda^{*}=\left(k^{*}\left(\frac{\delta(1-a)+\rho}{a}\right)\right)^{-1}
\end{array}\right.
$$

while $l^{*}$ is given by the resolution of the third equation in (2.2).

Next by setting $\mathrm{d}=\frac{\delta+\rho}{a}$ and $\nu=\frac{\mathrm{d}-\delta}{\mathrm{d}}$, the linearization problem in the neighbourhood of the homogeneous steady state yields the following linear problem

$$
\left\{\begin{aligned}
\frac{\partial u_{1}}{\partial t} & =\mathrm{d}\left[\left((\alpha-a) \phi_{1} * u_{1}+(a-1+\nu) u_{1}\right)+\nu u_{2}+(\beta-b) \phi_{2} * w+b w\right] \\
\frac{\partial u_{2}}{\partial t} & =-a d\left[(\alpha-a) * u_{1}+(a-1) u_{1}+(\beta-b) \phi_{2} * w+b w\right] \\
0 & =(\beta-b) \phi_{2} * w+(b+\chi-1) w+u_{2}+(\alpha-a) \phi_{1} * u_{1}+a u_{1}
\end{aligned}\right.
$$

The above system of equations, namely (2.4) is an illustrative example of linear non local differential-algebraic equation, for which the question of existence and uniqueness of a solution has to be tackled. To that aim, we shall present in the next subsection a more general non local differential-algebraic system of equations that will be studied in the sequel. The theoretical results obtained in this more general framework will then be illustrated in the study of Problem (2.4).

\subsection{The mathematical problem}

Motivated by the example described in the previous section, we study the existence of what we shall call below stable dichotomy for a class of the non local algebraic-differential system of equations of the form

$$
\frac{\partial}{\partial t}\left(\begin{array}{l}
u \\
0
\end{array}\right)=\left[\begin{array}{ll}
L_{11} & L_{12} \\
L_{21} & L_{22}
\end{array}\right]\left(\begin{array}{l}
u \\
w
\end{array}\right)
$$

where $u(\cdot, t)=\left(u_{1}, \ldots, u_{n_{1}}\right)^{T}(\cdot, t) \in L^{2}\left(\mathbb{R}^{p} ; \mathbb{R}^{n_{1}}\right), w(\cdot, t)=\left(w_{1}, \ldots, w_{n_{2}}\right)(\cdot, t) \in L^{2}\left(\mathbb{R}^{p} ; \mathbb{R}^{n_{2}}\right)$, for some integers $p \geq 1, n_{1} \geq 1$ and $n_{2} \geq 1$. Here, by setting the Hilbert spaces $H_{1}$ and $H_{2}$ defined by

$$
H_{j}=L^{2}\left(\mathbb{R}^{p}, \mathbb{R}^{n_{j}}\right), \quad j=1,2,
$$

\footnotetext{
${ }^{1}$ The Dirac delta function $\delta_{0}$ at $x=0$ is the probability measure defined for each Borel set $A$ as $\delta_{0}(A)=\left\{\begin{array}{l}1 \text { if } 0 \in A \\ 0 \text { else }\end{array}\right.$. Note also that it is a neutral element for the convolution product, that is $\delta_{0} * \phi=\phi * \delta_{0}=\phi$ for any integrable function $\phi$.
} 
the bounded linear operators $L_{i j} \in \mathcal{L}\left(H_{j}, H_{i}\right)$, for any $i, j=1,2$, take the following form

$$
L_{i j} \varphi(x)=A_{i j} \varphi(x)+\Phi_{i j} * \varphi(x) \forall \varphi \in H_{j},
$$

wherein $A_{i j}$ are $\left(n_{i} \times n_{j}\right)$ real matrices, that is $A_{i j} \in M_{n_{i}, n_{j}}(\mathbb{R})$ while the matrix valued function $\Phi_{i j}$ belong to $\Phi_{i j} \in L^{1}\left(\mathbb{R}^{p} ; M_{n_{i}, n_{j}}(\mathbb{R})\right)$.

In the above system the initial data $u(\cdot, 0)$ is partially known, in the sense that some components, say $u_{i}(\cdot, 0)$ for $i \in I \subset\left\{1, \ldots, n_{1}\right\}$, are prescribed while the other components $u_{j}(\cdot, 0)$ for $j \in\left\{1, \ldots, n_{1}\right\} \backslash I$ should be determined in such a way that, equipped with this initial data, Problem (2.5) admits a solution defined for all positive time.

To that aim and inspired by Arendt and Favini [3] we propose the following definition of integrated solution for Problem (2.5) supplemented with an initial data $u(\cdot, 0)=u_{0} \in H_{1}$ :

Definition 2.1. We say that a function pair $t \mapsto\left(u_{k}(t), W_{k}(t)\right) \in \mathcal{C}^{0}\left([0, \infty) ; H_{1} \times H_{2}\right)$ is a $k$-integrated solution of (2.5), for some integer $k \geq 1$, with the initial data $u_{0} \in H_{1}$ if it satisfies $W(0)=0$ and

$$
\left\{\begin{array}{l}
u_{k}(t)=\frac{t^{k-1}}{(k-1) !} u_{0}+L_{11} \int_{0}^{t} u_{k}(s) \mathrm{d} s+L_{12} W_{k}(t), \quad \forall t \geq 0 . \\
0=L_{21} \int_{0}^{t} u_{k}(s) \mathrm{d} s+L_{22} W_{k}(t),
\end{array}\right.
$$

Remark 2.2. In the above definition the function $u_{k}(t)$ and $W_{k}(t)$ stand for $\int_{0<s_{0}<\cdots<s_{k-1}<t} u\left(s_{0}\right) \mathrm{d} s_{0} \cdots \mathrm{d} s_{k-1}$ and $\int_{0<s_{0}<\cdots<s_{k}<t} w\left(s_{0}\right) \mathrm{d} s_{0} \cdots \mathrm{d} s_{k}$. However the function $W_{k}$ is not, in general, of the class $\mathcal{C}^{1}$ but, from the second equation, the function $t \mapsto L_{22} W_{k}(t)$ is of the class $\mathcal{C}^{1}$ with value in $H_{2}$.

Remark 2.3. One may also define a $k$-integrated solution of (2.5) backward in time as a pair of continuous function $\left(u_{k}(t), W_{k}(t)\right)$ defined for $t \geq 0$ and satisfying

$$
\left\{\begin{array}{l}
u_{k}(t)=\frac{t^{k-1}}{(k-1) !} u_{0}-L_{11} \int_{0}^{t} u_{k}(s) \mathrm{d} s-L_{12} W_{k}(t), \quad \forall t \geq 0 . \\
0=L_{21} \int_{0}^{t} u_{k}(s) \mathrm{d} s+L_{22} W_{k}(t),
\end{array}\right.
$$

Observe also that backward solutions correspond to solutions of the (backward) problem

$$
-\frac{\partial}{\partial t}\left(\begin{array}{l}
u \\
0
\end{array}\right)=\left[\begin{array}{ll}
L_{11} & L_{12} \\
L_{21} & L_{22}
\end{array}\right]\left(\begin{array}{c}
u \\
w
\end{array}\right)
$$

To study the solution of (2.5), we shall consider this problem in a $L^{2}$-functional framework. And, we shall determine a set of initial data leading to the existence of a solution of the forward and backward problem (2.5) and that satisfies the following behaviour as time goes to $\infty$ :

$$
\lim _{t \rightarrow \infty} u(\cdot, t)=0 \text { for the topology of } H_{1}=L^{2}\left(\mathbb{R}^{p} ; \mathbb{R}^{n_{1}}\right) .
$$

Observe that, on the contrary to the non-spatialized model, $w$ cannot be expressed explicitly as a function $u$. Some assumptions will have to be made on the operators which enter in the algebraic equation to ensure the existence of a solution of the algebraic equation. Moreover, as usual with Pontryagin's Maximum Principle methods, conditions on the initial data as a function of $L^{2}\left(\mathbb{R}^{p} ; \mathbb{R}^{n_{1}}\right)$ have to be found so that initial and terminal conditions are satisfied. 
The main tool to solve these questions relies on the Fourier transform. For any function $k$ in $L^{1}\left(\mathbb{R}^{p}\right)$ or $L^{2}\left(\mathbb{R}^{p}\right)$, we denote by $\widehat{k}$ or $\mathcal{F}(k)$ its Fourier transform that is defined (at least for integrable functions) by

$$
\widehat{k}(\xi)=\int_{\mathbb{R}^{p}} k(x) e^{-2 i \pi x \xi} \mathrm{d} x, \xi \in \mathbb{R}^{p} .
$$

We shall furthermore denote by $\mathcal{F}^{-1}$ the inverse Fourier transform on $L^{2}\left(\mathbb{R}^{p}\right)$. The same notation will be used for vector valued functions.

Using these notation, we consider, for any $i, j=1,2$, the continuous (matrix valued) functions $\xi \mapsto M_{i j}(\xi)$ defined by

$$
M_{i j}(\xi)=A_{i j}+\mathcal{F}\left(\Phi_{i j}\right)(\xi), \forall \xi \in \mathbb{R}^{p}, i, j=1,2,
$$

as well as the continuous function $D: \mathbb{R}^{p} \rightarrow \mathbb{C}$ defined by

$$
D(\xi)=\operatorname{det}\left(M_{22}(\xi)\right), \forall \xi \in \mathbb{R}^{p} .
$$

By formally applying the Fourier transform to (2.5) yields

$$
\frac{\partial}{\partial t}\left(\begin{array}{c}
\widehat{u}(\xi, t) \\
0
\end{array}\right)=\left[\begin{array}{ll}
M_{11}(\xi) & M_{12}(\xi) \\
M_{21}(\xi) & M_{22}(\xi)
\end{array}\right]\left(\begin{array}{l}
\widehat{u}(\xi, t) \\
\widehat{w}(\xi, t)
\end{array}\right) .
$$

Next, denoting by $N_{22}(\xi) \in M_{n_{2}, n_{2}}(\mathbb{C})$ the transposed of the matrix of the cofactors of $M_{22}(\xi)$, so that

$$
N_{22}(\xi) M_{22}(\xi)=M_{22}(\xi) N_{22}(\xi)=D(\xi) I_{n_{2}}, \forall \xi \in \mathbb{R}^{p}
$$

one obtains, from the second equation in (2.9), that

$$
D(\xi) \widehat{w}(\xi, t)=-N_{22}(\xi) M_{21}(\xi) \widehat{u}(\xi, t) .
$$

Multiplying the $\widehat{u}$-equation by $D(\xi)$ allows to eliminate $\widehat{w}$ and the resulting equation for $\widehat{u}$ reduces to the following degenerate equation

$$
\partial_{t}(D(\xi) \widehat{u}(\xi, t))=M(\xi) \widehat{u}(\xi, t) .
$$

Here we have defined the function $\xi \in \mathbb{R}^{p} \mapsto M(\xi) \in M_{n_{1}, n_{1}}(\mathbb{C})$ by

$$
M(\xi)=D(\xi) M_{11}(\xi)-M_{12}(\xi) N_{22}(\xi) M_{21}(\xi) .
$$

Recalling that all the matrix valued functions appearing in the above computations, namely $M_{i j}(\cdot), M(\cdot)$ and $N_{22}(\cdot)$, are uniformly bounded on $\mathbb{R}^{p}$, we consider the linear bounded operators $(\mathcal{A}, \mathcal{B}) \in \mathcal{L}\left(H_{1}\right)^{2}$ defined for any $\varphi \in H_{1}$ by

$$
\mathcal{A} \varphi=\mathcal{F}^{-1}(\xi \mapsto M(\xi) \widehat{\varphi}(\xi)) \text { and } \mathcal{B} \varphi=\mathcal{F}^{-1}(\xi \mapsto D(\xi) \widehat{\varphi}(\xi)) \text {. }
$$

Thus using these linear operators, Problem (2.5) formally re-writes as follows

$$
\frac{\mathrm{d}}{\mathrm{d} t}(\mathcal{B} u(t))=\mathcal{A} u(t) .
$$


This problem is supplemented with some initial data

$$
\mathcal{B} u(\cdot, 0)=\mathcal{B} u_{0}, \text { for some } u_{0} \in H_{1} .
$$

Note that since the function $\xi \mapsto D(\xi)$ may vanish, the operator $\mathcal{B}$ does not has a bounded inverse so that the above problem turns out to be degenerate.

In the sequel we shall provide sufficient conditions that will roughly ensure the existence of a splitting of the phase space $H_{1}=H_{1}^{s} \oplus H_{1}^{u}$ such that for each $u_{0} \in H_{1}^{s}$ (resp. $u_{0} \in H_{1}^{u}$ ) Problem (2.12) and (2.13) has a globally defined forward (resp. backward) solution. From this analysis we will be able to come back to the original problem (2.5).

\section{Existence of A DiChotomy SPLitting}

In this section, we discuss the existence of a decomposition of the state space $H_{1}$ into two closed subspaces, that will allow us to construct globally defined forward and backward solutions respectively for Problem (2.12) and (2.13). Using these information we shall then come back to the original problem (2.5) and we shall discuss the existence of suitable solutions for such a problem.

\subsection{Definition and main results}

According to Arendt and Favini [3] (see also the references cited therein), we define a notion of weak (or once integrated) solution for the above problem, namely (2.12) and (2.13).

Definition 3.1 (Weak solutions). A function $u \in \mathcal{C}^{0}\left([0, \infty) ; H_{1}\right)$ is said to be a forward weak solution of $(2.12)$ and (2.13) if it satisfies

$$
\mathcal{B} u(t)=\mathcal{B} u_{0}+\int_{0}^{t} \mathcal{A} u(s) \mathrm{d} s, \forall t \geq 0
$$

A function $u \in \mathcal{C}^{0}\left([0, \infty) ; H_{1}\right)$ is said to be a backward weak solution of (2.12) and (2.13) if it satisfies

$$
\mathcal{B} u(t)=\mathcal{B} u_{0}-\int_{0}^{t} \mathcal{A} u(s) \mathrm{d} s, \forall t \geq 0
$$

Such solutions will be refereed in the sequel as very weak solutions of (2.5).

Remark 3.2. Observe that if $t \mapsto u(t)$ is a forward weak solution then, since $\mathcal{A} \in \mathcal{L}\left(H_{1}\right)$, the map $t \mapsto$ $\mathcal{A} \int_{0}^{t} u(s) \mathrm{d} s$ is of the class $\mathcal{C}^{1}$ (as a $H_{1}$-valued function). Hence $t \mapsto \mathcal{B} u(t)$ is also of the class $\mathcal{C}^{1}$ and $t \mapsto u(t)$ is actually a strong solution of (2.12) and (2.13) in the sense that

$$
\frac{\mathrm{d}}{\mathrm{d} t}(\mathcal{B} u(t))=\mathcal{A} u(t), \forall t \geq 0
$$

The same remark also holds for backward solution. Observe also that backward solution corresponds to solution of the problem

$$
\frac{\mathrm{d}}{\mathrm{d} t}(\mathcal{B} u(t))=-\mathcal{A} u(t), \forall t \geq 0
$$

Our next definition deals with the notion of dichotomy for Problem (2.12). 
Definition 3.3 (Stable dichotomy). We say that Problem (2.12) admits a stable dichotomy if there exist two bounded projectors $\mathcal{P}^{s}, \mathcal{P}^{u} \in \mathcal{L}\left(H_{1}\right)$ and two strongly continuous semigroups $\left\{S^{s}(t)\right\}_{t \geq 0}$ and $\left\{S^{u}(t)\right\}_{t \geq 0}$ respectively on $H_{1}^{s}:=R\left(\mathcal{P}^{s}\right)$ and $H_{1}^{u}:=R\left(\mathcal{P}^{u}\right)$ such that the following set of properties are satisfied

(i) $\mathcal{P}^{s}+\mathcal{P}^{u}=1$,

(ii) $\mathcal{P}^{\alpha}(z-\mathcal{A})^{-1} \mathcal{B}=(z-\mathcal{A})^{-1} \mathcal{B} \mathcal{P}^{\alpha}$ for all $\alpha \in\{s, u\}$ and $z \in \rho(\mathcal{A})$,

(iii) For each $u_{0}^{s} \in H_{1}^{s}$ (resp. $u_{0}^{u} \in H_{1}^{u}$ ), the function $u^{s}(t)=S^{s}(t) u_{0}^{s}$ (resp. $u^{u}(t)=S^{u}(t) u_{0}^{u}$, defined for $t \geq 0$, is a forward (resp. a backward) weak solution of (2.12) with the initial data $u_{0}^{s}$ (resp. $u_{0}^{u}$ ).

(iv) The semigroups $S^{\alpha}$ satisfy

$$
\lim _{t \rightarrow \infty} S^{\alpha}(t) u_{0}^{\alpha}=0, \forall u_{0}^{\alpha} \in R\left(\mathcal{P}^{\alpha}\right), \alpha \in\{s, u\} .
$$

We now turn to the main assumptions that will be used in the sequel.

Assumption 3.4. We assume that the continuous function $D: \mathbb{R}^{p} \rightarrow \mathbb{C}$ defined in (2.8) satisfies the following properties:

(i) $D\left(\mathbb{R}^{p}\right) \subset \mathbb{R}$.

(ii) Its set of zeroes has a zero Lebesgue measure in $\mathbb{R}^{p}$, that is

$$
\operatorname{meas}\left(\left\{\xi \in \mathbb{R}^{p}: D(\xi)=0\right\}\right)=\operatorname{meas}\left(D^{-1}(\{0\})\right)=0 .
$$

Remark 3.5. Let us remark that the condition $(i)$ in the above assumption holds true in particular if for each $\xi \in \mathbb{R}^{p}$ the matrix $M_{22}(\xi)$ has real valued entries. Recalling that this matrix is constructed as the Fourier transform of the function $x \mapsto A_{22}+\Phi_{22}(x)$, condition $(i)$ is, in particular, ensured if the function $\Phi_{22}$ is symmetric, in the sense that $\Phi_{22}(-x)=\Phi_{22}(x)$ for all $x \in \mathbb{R}^{p}$.

In order to state our next set of assumptions, for each matrix $A \in M_{n_{1}, n_{1}}(\mathbb{C})$ we denote by $P(z, A)$ its characteristic polynomial, that is

$$
P(z, A)=\operatorname{det}\left(z I_{n_{1}}-A\right) .
$$

Next let us denote by $B_{22} \in M_{n_{2}, n_{2}}(\mathbb{R})$ the transposed of the matrix composed with the cofactors of the matrix $A_{22}$. We also set

$$
M^{\infty}:=\operatorname{det}\left(A_{22}\right) A_{11}-A_{12} B_{22} M_{21},
$$

so that $M(\xi) \rightarrow M^{\infty}$ as $\|\xi\| \rightarrow \infty$. With these notation we assume that:

Assumption 3.6. The continuous matrix $M: \mathbb{R}^{p} \rightarrow M_{n_{1}, n_{1}}(\mathbb{C})$ satisfies the following properties:

$$
\left\{\xi \in \mathbb{R}^{p}: \exists z \in i \mathbb{R}, P(z, M(\xi))=0\right\}=\left\{\xi_{1}, \ldots, \xi_{l}\right\}
$$

and, for each $k=1, \ldots, l$ one has

$$
\partial_{z} P\left(\zeta, M\left(\xi_{k}\right)\right) \neq 0, \forall \zeta \in\left\{z \in i \mathbb{R}: P\left(z, M\left(\xi_{k}\right)\right)=0\right\} .
$$

Moreover the matrix $M^{\infty}$ satisfies

$$
\partial_{z} P\left(\zeta, M^{\infty}\right) \neq 0, \forall \zeta \in\left\{z \in i \mathbb{R}: P\left(z, M^{\infty}\right)=0\right\} .
$$

We are now able to state our first main decomposition result. 
Theorem 3.7. Let Assumptions 3.4 and 3.6 be satisfied. Then Problem (2.12) admits a stable dichotomy on $H_{1}$, in the sense of Definition 3.3.

We continue this section by giving a strong link between the solutions of (2.12) constructed in Theorem 3.7 and those of Problem (2.5). It requires an extra assumption and our next result reads as follows.

Theorem 3.8. Let Assumptions 3.4 and 3.6 be satisfied. Assume furthermore that

$$
D\left(\xi_{j}\right) \neq 0, \forall j=1, \ldots, l \text { and } \operatorname{det} A_{22} \neq 0 .
$$

Let $u_{0}^{s} \in R\left(\mathcal{P}^{s}\right)$ and $u_{0}^{u} \in P\left(\mathcal{P}^{u}\right)$ be given. Then the functions $u^{s}: t \mapsto S^{s}(t) u_{0}^{s}$ and $u^{u}: t \mapsto S^{u}(t) u_{0}^{u}$ satisfy

$$
\int_{0}^{t} u^{\alpha}(l) \mathrm{d} l \in D\left(\mathcal{B}^{-1}\right), \forall t \geq 0, \alpha \in\{s, u\}
$$

Furthermore one has

$$
L_{21} \int_{0}^{t} u^{\alpha}(l) \mathrm{d} l \in R\left(L_{11}\right), \forall t \geq 0, \alpha=s, u
$$

The pair of function $\left(u^{s}, W^{s}\right)$, wherein $W^{s}(t)$ is defined by $W^{s}(t)=-L_{11}^{-1} L_{21} \int_{0}^{t} u^{s}(l) \mathrm{d} l$, satisfies $W^{s} \in$ $L_{\mathrm{loc}}^{\infty}\left([0, \infty) ; H_{2}\right)$ as well as the following system of equations, for all $t \geq 0$,

$$
\left\{\begin{array}{l}
u^{s}(t)=u_{0}^{s}+L_{11} \int_{0}^{t} u^{s}(l) \mathrm{d} l+L_{12} W^{s}(t) \\
0=L_{21} \int_{0}^{t} u^{s}(l) \mathrm{d} l+L_{11} W^{s}(t)
\end{array}\right.
$$

The pair of function $\left(u^{u}, W^{u}\right)$, wherein $W^{u}(t)$ is defined by $W^{u}(t)=-L_{11}^{-1} L_{21} \int_{0}^{t} u^{u}(l) \mathrm{d} l$, satisfies $W^{u} \in$ $L_{\mathrm{loc}}^{\infty}\left([0, \infty) ; H_{2}\right)$ and the following system of equations, for all $t \geq 0$,

$$
\left\{\begin{array}{l}
u^{u}(t)=u_{0}^{u}-L_{11} \int_{0}^{t} u^{u}(l) \mathrm{d} l-L_{12} W^{u}(t), \\
0=L_{21} \int_{0}^{t} u^{u}(l) \mathrm{d} l+L_{11} W^{u}(t) .
\end{array}\right.
$$

Note that the above result does not ensure the existence of (once) integrated forward or backward solution of (2.5) since the functions $W^{\alpha}$ are not necessarily continuous in time. However, as a direct corollary of the above result, by integrating $\left(u^{\alpha}, W^{\alpha}\right)$ in time - so that the functions $t \mapsto \int_{0}^{t} W^{\alpha}(l) \mathrm{d} l$ become continuous - we obtain the following corollary.

Corollary 3.9. Under the assumptions of Theorem 3.8, for each initial data $u_{0}^{s} \in H^{s}$ and $u_{0}^{u} \in H^{u}$ respectively, Problem (2.5) has a 2-integrated forward (respectively backward) solution, denoted by $\left(u_{2}^{s}, W_{2}^{s}\right)$ and $\left(u_{2}^{u}, W_{2}^{u}\right)$ respectively, and associated with the initial data $u_{0}^{s}$ and $u_{0}^{u}$ respectively. Moreover $u_{2}^{\alpha}(t) \in H_{1}^{\alpha}$ for any $t \geq 0$ and $\alpha \in\{s, u\}$.

\subsection{Proofs of Theorem 3.7 and Theorem 3.8}

This section is devoted to the proof of the results stated in the previous section.

We are firstly concerned with the proof of Theorem 3.7. To that aim, recall that the function $\xi \mapsto M(\xi)$ is defined in (2.10). For each $\xi \in \mathbb{R}^{p}$ we denote by $\Pi^{c s}(\xi)$ and $\Pi^{c u}(\xi)$ the center stable and the center unstable spectral projector of the matrix $M(\xi)$. They respectively correspond to projector on the centre stable and centre 
unstable spectrum, that is on the spectral sets $\sigma^{c s}(M(\xi)):=\sigma(M(\xi)) \cap\{z \in \mathbb{C}: \operatorname{Re}(z) \leq 0\}$ and $\sigma^{c u}(M(\xi)):=$ $\sigma(M(\xi)) \cap\{z \in \mathbb{C}: \operatorname{Re}(z) \geq 0\}$. Let us observe that, due to Assumption 3.4, one has

$$
\sigma^{c u}(M(\xi)) \cap \sigma^{c u}(M(\xi)) \neq \emptyset \Leftrightarrow \xi \in\left\{\xi_{1}, \ldots, \xi_{l}\right\} .
$$

The proof of Theorem 3.7 will follow from the following result:

Proposition 3.10. Let Assumptions 3.4 and 3.6 be satisfied. Then the following hold true: the spectral projectors $\Pi^{c s}$ and $\Pi^{c u}$ are measurable and bounded on $\mathbb{R}^{p}$ and one has

$$
\sup _{t \geq 0, \xi \in \mathbb{R}^{p}}\left\|e^{M(\xi) t} \Pi^{c s}(\xi)\right\|<\infty, \text { and } \sup _{t \leq 0, \xi \in \mathbb{R}^{p}}\left\|e^{M(\xi) t} \Pi^{c u}(\xi)\right\|<\infty .
$$

In order to prove this proposition let us introduce some notation. For each set $I \subset \mathbb{R}$ we denote by $S(I) \subset \mathbb{C}$ the strip defined by

$$
S(I)=\{z \in \mathbb{C}: \operatorname{Re}(z) \in I\} .
$$

In the following we shall also denote by $N(z, \xi) \in M_{n_{1}}(\mathbb{C})$ the transposed co-matrix (matrix of the cofactors) of the matrix $z I_{n_{1}}-M(\xi)$, so that we get the following identity:

$$
\left(z I_{n_{1}}-M(\xi)\right) N(z, \xi)=P(z, M(\xi)) I_{n_{1}}, \forall z \in \mathbb{C}, \xi \in \mathbb{R}^{p} .
$$

Hence for all $\xi \in \mathbb{R}^{p}$ and $z \in \rho(M(\xi))$ (the resolvent set of $M(\xi)$ ) we have

$$
\left(z I_{n_{1}}-M(\xi)\right)^{-1}=\frac{1}{P(z, M(\xi))} N(z, \xi) .
$$

Next, since the entries of the matrix $M(\xi)$ are uniformly bounded with respect to $\xi \in \mathbb{R}^{p}$, we fix a value $R>0$ such that

$$
\sigma(M(\xi)) \cup \sigma\left(M^{\infty}\right) \subset D(0, R)
$$

Here we use the notation $D(0, R)$ to denote the open disc in $\mathbb{C}$ centred at $z=0$ with radius $R$ while the notation $B\left(\xi_{0}, \rho\right) \subset \mathbb{R}^{p}$ will be used to denote the open ball in $\mathbb{R}^{p}$ with center $\xi_{0} \in \mathbb{R}^{p}$ and radius $\rho>0$.

Now the proof of the above proposition relies on the following separation lemma:

Lemma 3.11. Under Assumptions 3.4 and 3.6, there exist $\eta>0$ and $\varepsilon>0$ small enough such that the following properties hold true.

(i) For all $\xi \in \mathbb{R}^{p}$ and $\lambda \in S([-\eta, \eta])$ one has

$$
P(\lambda, M(\xi))=0 \Rightarrow\left|\partial_{z} P(\lambda, M(\xi))\right| \geq \varepsilon ;
$$

(ii) for all $\xi \in \mathbb{R}^{p}, \lambda_{1}, \lambda_{2} \in S([-\eta, \eta])$ one has

$$
P\left(\lambda_{i}, M(\xi)\right)=0 \text { and } \lambda_{1} \neq \lambda_{2} \Rightarrow\left|\lambda_{1}-\lambda_{2}\right| \geq \varepsilon ;
$$

(iii) for all $\xi \in \mathbb{R}^{p},\left(\lambda_{1}, \lambda_{2}\right) \in S((-\eta, \eta)) \times[S((-\infty, \eta]) \cup S([\eta, \infty))]$ one has

$$
P\left(\lambda_{i}, M(\xi)\right)=0 \Rightarrow\left|\lambda_{1}-\lambda_{2}\right| \geq \varepsilon .
$$


Proof of Lemma 3.11. This proof is split into three parts corresponding to the three points stated in the above lemma.

Proof of $(i)$ : To prove this point let us argue by contradiction by assuming that there exist a sequence $\left\{\eta_{n}\right\}_{n} \geq 0$ such that $\eta_{n}>0$ and $\eta_{n} \rightarrow 0$ as $n \rightarrow \infty$, a sequence $\left\{\lambda_{n}\right\}_{n \geq 0}$ and a sequence $\left\{\xi_{n}\right\}_{n \geq 0} \subset \mathbb{R}^{p}$ such that for all $n \geq 0$

$$
\left.\lambda_{n} \in S\left(\left[-\eta_{n}, \eta_{n}\right]\right), P\left(\lambda_{n}, M\left(\xi_{n}\right)\right)=0 \text { and } \lim _{n \rightarrow \infty} \partial_{z} P\left(\lambda_{n}, M\left(\xi_{n}\right)\right)\right)=0 .
$$

Since $\lambda_{n} \in D(0, R)$ (see (3.6)) and $\eta_{n} \rightarrow 0$, one may assume, possibly along a subsequence, that $\lambda_{n} \rightarrow \lambda_{\infty} \in i \mathbb{R}$.

We now split the argument into two parts according to either $\left\{\xi_{n}\right\}_{n \geq 0}$ is bounded or unbounded.

If $\left\{\xi_{n}\right\}_{n \geq 0}$ is bounded, then we assume, possibly along a subsequence, that $\xi_{n} \rightarrow \xi_{\infty} \in \mathbb{R}$. Then passing to the limit into (3.7) yields

$$
\left.\lambda_{\infty} \in i \mathbb{R}, P\left(\lambda_{\infty}, M\left(\xi_{\infty}\right)\right)=0 \text { and } \partial_{z} P\left(\lambda_{\infty}, M\left(\xi_{\infty}\right)\right)\right)=0
$$

a contradiction with Assumption 3.6.

If the sequence $\left\{\xi_{n}\right\}_{n \geq 0}$ is unbounded, then one may assume that $\left\|\xi_{n}\right\| \rightarrow \infty$ as $n \rightarrow \infty$. Then the same arguments as above yield

$$
\left.\lambda_{\infty} \in i \mathbb{R}, P\left(\lambda_{\infty}, M^{\infty}\right)=0 \text { and } \partial_{z} P\left(\lambda_{\infty}, M^{\infty}\right)\right)=0 .
$$

This also provides a contradiction with Assumption 3.6 and completes the proof of $(i)$.

Proof of $($ ii $)$. In order to prove (ii) we still argue by contradiction by assuming there exist a sequence $\left\{\eta_{n}\right\}_{n \geq 0}$ tending to 0 , a sequence $\left\{\xi_{n}\right\}_{n \geq 0} \subset \mathbb{R}^{p}$ and two sequences $\lambda_{1, n} \neq \lambda_{2, n} \in S\left(\left[-\eta_{n}, \eta_{n}\right]\right)$ such that

$$
P\left(\lambda_{j, n}, M\left(\xi_{n}\right)\right)=0 \text { with } j=1,2 \text { and }\left|\lambda_{1, n}-\lambda_{2, n}\right| \rightarrow 0 .
$$

Since $\eta_{n} \rightarrow 0$, possibly along a subsequence, one may assume that

$$
\lim _{n \rightarrow \infty} \lambda_{j, n}=\lambda_{\infty} \in i \mathbb{R}, \forall j=1,2 .
$$

Next, as in the proof of $(i)$ we split the argument into two parts according to the boundedness of the sequence $\left\{\xi_{n}\right\}_{n \geq 0}$.

First case: if $\left\{\xi_{n}\right\}_{n \geq 0}$ is bounded, then one may assume that $\xi_{n} \rightarrow \xi_{\infty} \in \mathbb{R}^{p}$. On the other hand one gets for all $z \in \mathbb{C}$

$$
P\left(z, M\left(\xi_{n}\right)\right)=\prod_{j=1}^{2}\left(z-\lambda_{j, n}\right) \times Q_{n}(z) \rightarrow P\left(z, M\left(\xi_{\infty}\right)\right)=\left(z-\lambda_{\infty}\right)^{2} Q_{\infty}(z),
$$

wherein $Q_{n}(z)$ and $Q(z)$ are polynomials. Hence this yields

$$
\lambda_{\infty} \in i \mathbb{R}, P\left(\lambda_{\infty}, M\left(\xi_{\infty}\right)\right)=0 \text { and } \partial_{z} P\left(\lambda_{\infty}, M\left(\xi_{\infty}\right)\right)=0,
$$

a contradiction together with Assumption 3.6.

Second case: If $\left\{\xi_{n}\right\}_{n \geq 0}$ is unbounded then one may assume that $\left\|\xi_{n}\right\| \rightarrow \infty$ as $n \rightarrow \infty$. Then the same arguments as above apply with $M\left(\xi_{\infty}\right)$ replaced by $M^{\infty}$ (see (3.1)). And, this completes the proof of $(i i)$.

Proof of $(i i i)$. Here again we argue by contradiction and the proof of (iii) is similar to the proof of $(i i)$. The details are omitted and left to the reader. 
Proof of Proposition 3.10. We split this proof into two main steps. We first prove the regularity, namely measurability, of the spectral projector. In a second step, we prove the boundedness of $e^{M(\xi) t} \Pi^{c s}(\xi)$ with respect to $t \geq 0$ and $\xi \in \mathbb{R}^{p}$. The boundedness of the center unstable part follows the same lines.

Regularity of the projectors. To prove that the center stable spectral projector is measurable, we investigate the two cases: around a point $\xi_{0}$ where the spectrum is far from the imaginary axis and around some point $\xi_{j}$ (see Assumption 3.6) where the spectrum may cross the imaginary axis.

Let $\xi_{0} \in \mathbb{R}^{p}$ be given such that $\sigma\left(M\left(\xi_{0}\right)\right) \cap i \mathbb{R}=\emptyset$, that is $\min _{z \in i \mathbb{R}}\left|P\left(z, M\left(\xi_{0}\right)\right)\right|>0$. Then by the continuity of the function $(z, \xi) \mapsto P(z, M(\xi))$, there exists $\nu>0$ small enough such that

$$
\min _{\xi \in \bar{B}\left(\xi_{0}, \nu\right), z \in i \mathbb{R}}|P(z, M(\xi))|>0 .
$$

This, in particular, ensures that for all $\xi \in B\left(\xi_{0}, \nu\right)$ one has

$$
\sigma^{c s}(M(\xi))=\sigma(M(\xi)) \cap S((-\infty, 0)) \text { and } \inf _{\xi \in B\left(\xi_{0}, \nu\right)} \operatorname{dist}\left(\sigma^{c s}(M(\xi)), i \mathbb{R}\right)>0
$$

Recalling the definition of $R>0$ in (3.6), one gets for all $\xi \in B\left(\xi_{0}, \nu\right)$

$$
\Pi^{c s}(\xi)=\frac{1}{2 i \pi} \oint_{\gamma} N(z, \xi) \frac{\mathrm{d} z}{P_{M}(z, \xi)},
$$

where $\gamma$ denotes the continuous and piecewise smooth contour given by the boundary of the domain $[D(0, R+1) \cap S((-\infty, 0])]$. In the above formula, $N(z, \xi)$ is given in (3.5). Hence, using the above formula for the spectral projector, we deduce that the map $\mapsto \Pi^{c s}(\xi)$ is continuous at $\xi=\xi_{0}$.

Now we discuss the regularity of $\Pi^{c s}(\xi)$ around $\xi=\xi_{j}$, for some fixed $j \in\{1, \ldots, l\}$ as defined in Assumption 3.6. To that aim we write, for some integer $p_{j} \in\left\{1, \ldots, n_{1}\right\}$,

$$
\left\{z \in i \mathbb{R}: P_{M}\left(z, \xi_{j}\right)=0\right\}=\left\{\zeta_{1}, \ldots, \zeta_{p_{j}}\right\}
$$

Due to Lemma 3.11 and the implicit function theorem (see [33], Thm. 9.3), the exist $\nu>0$ small enough, $0<\bar{\eta}<\eta$ small enough and $p_{j}$ continuous functions $z_{k}: B\left(\xi_{j}, \nu\right) \rightarrow S((-\bar{\eta}, \bar{\eta}))$ such that $z_{k}\left(\xi_{j}\right)=\zeta_{k}$ for all $k=1, \ldots, p_{j}$ and such that, for all $\xi \in B\left(\xi_{j}, \nu\right)$ one has

$$
\{z \in S((-\bar{\eta}, \bar{\eta})): P(z, M(\xi))=0\}=\left\{z_{1}(\xi), \ldots, z_{p_{j}}(\xi)\right\}
$$

Once again the application of Lemma 3.11 (ii) ensures that for each $\xi \in B\left(\xi_{j}, \nu\right)$, the spectral projector, denoted by $\Pi^{\bar{\eta}}(\xi)$, on the strip $S(-\bar{\eta}, \bar{\eta})$ can be written, using residues theorem, as

$$
\Pi^{\bar{\eta}}(\xi)=\sum_{k=1}^{p_{j}} \frac{1}{2 i \pi} \oint_{\partial D\left(z_{k}(\xi), \frac{\varepsilon}{2}\right)} N(z, \xi) \frac{\mathrm{d} z}{P(z, M(\xi))}=\sum_{k=1}^{p_{j}} \frac{N\left(z_{k}(\xi), \xi\right)}{\partial_{z} P\left(z_{k}(\xi), M(\xi)\right)} .
$$

Next, up to reduce $\nu$ if necessary, one may assume that $\sigma(M(\xi)) \cap S\left(\left(-\bar{\eta},-\frac{\bar{\eta}}{2}\right)\right)=\emptyset$ for all $\xi \in B\left(\xi_{j}, \nu\right)$. Hence for any $\xi \in B\left(\xi_{j}, \nu\right), \Pi^{c s}(\xi)$ can be written as follows

$$
\Pi^{c s}(\xi)=\Pi^{\mathrm{far}}(\xi)+\sum_{k=1}^{p_{j}} \chi_{\left\{\operatorname{Re}\left(z_{k}(\xi)\right) \leq 0\right\}} \frac{N\left(z_{k}(\xi), \xi\right)}{\partial_{z} P\left(z_{k}(\xi), M(\xi)\right)},
$$


wherein the operator $\Pi^{\mathrm{far}}(\xi)$ is defined as the spectral projector on $\sigma(M(\xi)) \cap S\left(\left(-\infty,-\frac{\bar{\eta}}{2}\right]\right)$. It is furthermore explicitly defined by

$$
\Pi^{\mathrm{far}}(\xi)=\frac{1}{2 i \pi} \oint_{\gamma} N(z, \xi) \frac{\mathrm{d} z}{P_{M}(z, \xi)}
$$

with $\gamma$ denotes a continuous and piecewise smooth parametrisation of the boundary of the domain $\left[D(0, R+1) \cap S\left(\left(-\infty,-\frac{3 \bar{\eta}}{4}\right)\right)\right]$.

Since $\Pi^{\text {far }}$ is continuous at $\xi=\xi_{j}$, the map $\xi \mapsto \Pi_{\mid B\left(x_{j}, \nu\right)}^{c s}(\xi)$ becomes measurable. Finally because of the finite condition in Assumption 3.6 and the continuity property proved above, the function $\Pi^{c s}$ is therefore measurable on $\mathbb{R}^{p}$. The same arguments apply to study the regularity of the spectral projector on the center unstable spectrum.

Boundedness. In order to prove the boundedness properties as stated in Proposition 3.10, let $\eta>0$ and $0<\varepsilon<\eta$ be the constants provided by Lemma 3.11. Next for each given $\xi \in \mathbb{R}^{p}$, consider the spectral sets $A(\xi)$ and $B(\xi)$ defined by

$$
A(\xi)=\sigma(M(\xi)) \cap S((-\infty,-\eta]) \text { and } B(\xi)=\sigma(M(\xi)) \cap S((-\eta, 0]) .
$$

Denote by $\Pi^{A}(\xi)$ and $\Pi^{B}(\xi)$ respectively the spectral projectors on the spectral sets $A(\xi)$ and $B(\xi)$ respectively. Obverse that

$$
\Pi^{c s}(\xi)=\Pi^{A}(\xi)+\Pi_{B}(\xi) \text { for all } \xi \in \mathbb{R}^{p} .
$$

Note also that due to Lemma 3.11 (iii) one has

$$
\operatorname{dist}(A(\xi), B(\xi)) \geq \varepsilon, \forall \xi \in \mathbb{R}^{p} .
$$

In order to investigate the uniform boundedness, with respect to $\xi \in \mathbb{R}^{p}$ and $t \geq 0$, of the operator $e^{M(\xi) t} \Pi^{c s}(\xi)$, we consider the two operators $e^{M(\xi) t} \Pi^{A}(\xi)$ and $e^{M(\xi) t} \Pi^{B}(\xi)$ separately.

The case of $B(\xi)$. Fix $\xi \in \mathbb{R}^{p}$ such that $B(\xi) \neq \emptyset$ and let us write $B(\xi)=\left\{z_{1}(\xi), \ldots, z_{p(\xi)}(\xi)\right\}$ for some $p(\xi) \in\left\{1, \ldots, n_{1}\right\}$. Note that if $B(\xi)=\emptyset$ then $\Pi^{B}(\xi)=0$ and the proof is already over.

Due to Lemma $3.11(\mathrm{ii})$, the following representation holds true:

$$
\Pi^{B}(\xi)=\sum_{k=1}^{p(\xi)} \frac{1}{2 i \pi} \oint_{\partial D\left(z_{k}(\xi), \frac{\varepsilon}{2}\right)} N(z, \xi) \frac{\mathrm{d} z}{P(z, M(\xi))}
$$

Using Lemma $3.11(i)$ and residues theorem, one gets

$$
\Pi^{B}(\xi)=\sum_{k=1}^{p(\xi)} N\left(z_{k}(\xi), \xi\right) \frac{1}{\partial_{z} P\left(z_{k}(\xi), M(\xi)\right)} .
$$

Hence for all $\xi \in \mathbb{R}^{p}$ one gets.

$$
\left\|\Pi^{B}(\xi)\right\|_{\mathcal{L}\left(H_{1}\right)} \leq \frac{n_{1}}{\varepsilon} \sup _{|z| \leq \mathbb{R}, \widetilde{\xi} \in \mathbb{R}^{p}}\|N(z, \widetilde{\xi})\|
$$


Moreover one has

$$
e^{M(\xi) t} \Pi^{B}(\xi)=\sum_{k=1}^{p(\xi)} e^{z_{k}(\xi) t} N\left(z_{k}(\xi), \xi\right) \frac{1}{\partial_{z} P\left(z_{k}(\xi), M(\xi)\right)} .
$$

As a consequence, since $\operatorname{Re}\left(z_{k}(\xi)\right) \leq 0$, we obtain that $e^{M(\xi) t} \Pi^{B}(\xi)$ is uniformly bounded with respect to $t \geq 0$ and $\xi \in \mathbb{R}^{p}$.

The case of $A(\xi)$. Fix $\xi \in \mathbb{R}^{p}$ such that $A(\xi) \neq \emptyset$. Next if $\sigma(M(\xi)) \cap S((-\eta, \eta)) \neq \emptyset$, let us write for some $m(\xi) \in\left\{1, \ldots, n_{1}\right\}$,

$$
\sigma(M(\xi)) \cap S((-\eta, \eta))=\left\{\lambda_{1}(\xi), \ldots, \lambda_{m(\xi)}(\xi)\right\} .
$$

And, let us consider the set $\Omega(\xi)$ defined by

$$
\Omega(\xi)=\left\{\begin{array}{l}
(D(0, R+1) \cap S((-\infty, 0])) \text { if } \sigma(M(\xi)) \cap S((-\eta, \eta)) \neq \emptyset \\
(D(0, R+1) \cap S((-\infty, 0])) \backslash\left(\cup_{k=1}^{m(\xi)} D\left(\lambda_{k}(\xi), \frac{\varepsilon}{2}\right)\right) \text { else. }
\end{array}\right.
$$

Note that each of the finite number of the connected components of the boundary $\partial \Omega(\xi)$ are continuous and piecewise smooth. Moreover the length of this boundary, denoted by $\operatorname{Per}(\Omega(\xi))$, is uniformly bounded with respect to $\xi \in \mathbb{R}^{p}$.

Now observe that, due to Lemma 3.11 (ii) and (iii), one has

$$
\min _{z \in \partial \Omega(\xi)}|P(z, M(\xi))| \geq\left(\frac{\varepsilon}{2}\right)^{n_{1}}, \forall \xi \in \mathbb{R}^{p}
$$

Next note also that it holds that

$$
\Pi^{A}(\xi)=\frac{1}{2 i \pi} \oint_{\partial \Omega(\xi)} N(z, \xi) \frac{\mathrm{d} z}{P_{M}(z, \xi)}, \forall \xi \in \mathbb{R}^{p}
$$

We firstly conclude that

$$
\left\|\Pi^{A}(\xi)\right\|_{\mathcal{L}\left(H_{1}\right)} \leq\left(\frac{2}{\varepsilon}\right)^{n_{1}} \frac{1}{2 \pi} \sup _{z \in D(0, R+1), \widetilde{\xi} \in \mathbb{R}^{p}}\|N(z, \widetilde{\xi})\| \times \operatorname{Per}(\Omega(\xi)) .
$$

Hence because of the above remarks, the function $\xi \mapsto \Pi^{A}(\xi)$ is uniformly bounded on $\mathbb{R}^{p}$. Moreover observe also that the spectral bound of the operator $M(\xi) P^{A}(\xi)$ is less that $-\eta<0$. Hence Coppel's lemma (see Lem. A.1) applies and ensures that $e^{M(\xi) t} \Pi^{A}(\xi)$ is uniformly bounded with respect to $t \geq 0$ and $\xi \in \mathbb{R}^{p}$.

Finally, recalling that $\Pi^{c s}=\Pi^{A}+\Pi^{B}$ completes the proof of Proposition 3.10 for the center stable part. The proof for the center unstable part is similar. Thus it is omitted.

Equipped with Proposition 3.10 we are able to complete the proof of Theorem 3.7.

Proof of Theorem 3.7. The proof of Theorem 3.7 directly follows from Proposition 3.10. Note that for each $\varphi \in H_{1}$ the function $\xi \mapsto\left[\Pi^{c s}(\xi) \chi_{\{D(\xi)>0\}}+\Pi^{c u}(\xi) \chi_{\{D(\xi)<0\}}\right] \widehat{\varphi}(\xi)$ belongs to $H_{1}$. Next set

$$
\mathcal{P}^{s} \varphi:=\mathcal{F}^{-1}\left(\xi \mapsto\left[\Pi^{c s}(\xi) \chi_{\{D(\xi)>0\}}+\Pi^{c u}(\xi) \chi_{\{D(\xi)<0\}} \widehat{\varphi}(\xi)\right) \text { and } \mathcal{P}^{u}=I-\mathcal{P}^{s}\right.
$$


Because of the boundedness properties stated in Proposition 3.10, these two linear operators are bounded on $H_{1}$. Note also that (3.4) ensures that they are projectors on $H_{1}$, namely $\mathcal{P}^{\alpha} \circ \mathcal{P}^{\alpha}=\mathcal{P}^{\alpha}$ for any $\alpha \in\{s, u\}$. Furthermore from their construction these projectors commute both with the linear operator $\mathcal{B}$ and $\mathcal{A}$.

We also define for any $t \geq 0$ the linear operators $S^{\alpha}(t)$ for $\alpha \in\{s, u\}$ on $H_{1}^{\alpha}:=R\left(\mathcal{P}^{\alpha}\right)$ by

$$
S^{\alpha}(t) \mathcal{P}^{\alpha} \varphi=\mathcal{F}^{-1}\left(\xi \mapsto \exp \left(\varepsilon_{\alpha} \frac{t}{D(\xi)} M(\xi)\right) \widehat{\mathcal{P}^{\alpha} \varphi}(\xi)\right) .
$$

Here we have set

$$
\varepsilon_{\alpha}= \begin{cases}1 & \text { if } \alpha=s \\ -1 & \text { if } \alpha=u\end{cases}
$$

Let us observe that these two families of linear operator form both strongly continuous semigroups on $H_{1}^{\alpha}$. It also follows, from their constructions, that for each $u_{0}^{\alpha} \in H_{1}^{\alpha}$ with $\alpha=s, u$, the maps $t \mapsto S^{s}(t) u_{0}^{s}$ and $t \mapsto S^{u}(-t) u_{0}^{u}$ defined on $[0, \infty)$ are respectively forward and backward solution of $(2.12)$.

In addition since one has for almost every $\xi \in \mathbb{R}^{p}$,

$$
\lim _{t \rightarrow \infty} e^{M(\xi) t} \Pi^{c s}(\xi)=0 \text { and } \lim _{t \rightarrow-\infty} e^{M(\xi) t} \Pi^{c u}(\xi)=0,
$$

Lebesgue convergence theorem applies and ensures that for all $\alpha \in\{s, u\}$ and all $\varphi \in R\left(\mathcal{P}^{\alpha}\right)$ one has

$$
\lim _{t \rightarrow \infty} S^{\alpha}(t) \varphi=0 \text { in } H_{1} .
$$

This completes the proof of Theorem 3.7.

We are now able to prove Theorem 3.8 .

Proof of Theorem 3.8. The proof of this result is based on the following claim:

Claim 3.12. There exist $\eta>0$ and $\rho>0$ such that for all $\xi^{*} \in D^{-1}(\{0\})$ one has

$$
\sigma(M(\xi)) \cap S([-\eta, \eta])=\emptyset, \forall \xi \in B\left(\xi^{*}, \rho\right) .
$$

Proof. To prove this claim we argue by contradiction by assuming that there exist two sequences $\left\{\eta_{n}\right\}_{n \geq 0}$ and $\left\{\rho_{n}\right\}_{n \geq 0}$ tending to zero, a sequence $\left\{\xi_{n}^{*}\right\}_{n \geq 0} \in D^{-1}(\{0\})$, a sequence $\left\{\xi_{n}\right\}_{n \geq 0}$ and a sequence $\left\{\lambda_{n}\right\}_{n \geq 0}$ such that for all $n \geq 0$ :

$$
\xi_{n} \in B\left(\xi_{n}, \rho_{n}\right) \text { and } \lambda_{n} \in S\left(\left[-\eta_{n}, \eta_{n}\right]\right)
$$

and

$$
P\left(\lambda_{n}, M\left(\xi_{n}\right)\right)=0, \forall n \geq 0 .
$$

Up to a subsequence and since $D^{-1}(\{0\})$ is compact (see the second condition in (3.2)), we assume that

$$
\lambda_{n} \rightarrow \lambda_{\infty} \in i \mathbb{R} \text { and } \xi_{n}^{*} \rightarrow \xi_{\infty}^{*} \text { as } n \rightarrow \infty .
$$


Next observe that, since $\rho_{n} \rightarrow 0$, one also has $\xi_{n} \rightarrow \xi_{\infty}^{*}$ as $n \rightarrow \infty$. Hence we get

$$
D\left(\xi_{\infty}^{*}\right)=0, \lambda_{\infty} \in i \mathbb{R} \text { and } P\left(\lambda_{\infty}, M\left(\xi_{\infty}^{*}\right)\right)=0
$$

a contradiction with (3.2).

Now to complete the proof of Theorem 3.8, let us observe that due to Coppel's lemma (see Lem. A.1), there exist two constants $C>0$ and $\alpha>0$ such that for all $t \geq 0$

$$
\left\|e^{M(\xi) t} \Pi^{c s}(\xi)\right\|+\left\|e^{-M(\xi) t} \Pi^{c u}(\xi)\right\| \leq C e^{-\alpha t}, \forall \xi \in \bigcup_{\xi^{*} \in D^{-1}(\{0\})} B\left(\xi^{*}, \rho\right) .
$$

Furthermore, due to the second part of Condition (3.2), there exists $\varepsilon>0$ such that

$$
|D(\xi)| \geq \varepsilon, \forall \xi \in \mathbb{R}^{p} \backslash\left(\bigcup_{\xi^{*} \in D^{-1}(\{0\})} B\left(\xi^{*}, \rho\right)\right) .
$$

Now let us observe that the above estimates allow us to obtain the following upper estimate for any $t \geq 0$ :

$$
\begin{aligned}
& \left\|\int_{0}^{t} \frac{1}{D(\xi)} e^{M(\xi) \frac{s}{D(\xi)}} \mathrm{d} s\left[\Pi^{c s}(\xi) \chi_{\{D(\xi)>0\}}+\Pi^{c u}(\xi) \chi_{\{D(\xi)<0\}}\right]\right\| \\
& \leq\left\|\int_{0}^{\frac{t}{D(\xi)}} e^{M(\xi) l} \mathrm{~d} l\left[\Pi^{c s}(\xi) \chi_{\{D(\xi)>0\}}+\Pi^{c u}(\xi) \chi_{\{D(\xi)<0\}}\right]\right\| \\
& \leq M \frac{t}{\varepsilon}+2 C \int_{0}^{\infty} e^{-\alpha l} \mathrm{~d} l,
\end{aligned}
$$

wherein $M>0$ denotes the upper bound provided by Proposition 3.10.

As a consequence, recalling the definition of the linear operator $\mathcal{B}$ in (2.11), one gets that (3.3) holds true. Hence Theorem 3.8 directly follows for any initial data in $H_{1}^{s}$. The case of initial data in $H_{1}^{u}$ follows from similar arguments. This completes the proof of Theorem 3.8 .

\subsection{Further results in a periodic setting}

In this section, we revisit the properties of system (2.5) in a periodic context. Our motivation is twofold. First, many economic papers have considered periodic space domains [9, 23]. Moreover, within this context, the invariant manifolds are stable with respect to small perturbations, which enables to derive properties for the nonlinear initial model. More precisely, with the same notations as the above previous section, we consider

$$
\frac{\partial}{\partial t}\left(\begin{array}{l}
u \\
0
\end{array}\right)=\left[\begin{array}{ll}
L_{11} & L_{12} \\
L_{21} & L_{22}
\end{array}\right]\left(\begin{array}{c}
u \\
w
\end{array}\right)
$$

where $u(\cdot, t)=\left(u_{1}, \ldots, u_{n_{1}}\right)^{T}(\cdot, t) \in H_{\text {per }}^{k}\left(\mathbb{R}^{p} ; \mathbb{R}^{n_{1}}\right), w(\cdot, t)=\left(w_{1}, \ldots, w_{n_{2}}\right)(\cdot, t) \in H_{\text {per }}^{k}\left(\mathbb{R}^{p} ; \mathbb{R}^{n_{2}}\right)$, for some integers $p \geq 1, n_{1} \geq 1$ and $n_{2} \geq 1$ and where $k>0$ is a given and fixed real number while the subscript per is used for 1-periodic (in all directions) functions and $H^{k}$ denotes the usual Sobolev space. Here, by setting the Hilbert spaces $H_{1}$ and $H_{2}$ defined by

$$
H_{j}=H_{\mathrm{per}}^{k}\left(\mathbb{R}^{p}, \mathbb{R}^{n_{j}}\right), j=1,2,
$$


the operators $L_{i, j}$ are bounded from $H_{j}$ into $H_{i}$. To study the above problem in this periodic setting one introduce the Fourier coefficients for $k \in L_{\mathrm{per}}^{1}\left(\mathbb{R}^{p}\right)$ as

$$
c_{n}[k]=\int_{[0,1]^{p}} k(x) e^{-2 i \pi n x} \mathrm{~d} x, \forall n \in \mathbb{Z}^{p} .
$$

Then, one may reproduce similar computations as in the previous section, to obtain the following lemma

Lemma 3.13 (Invertibility of $L_{22}$ ). With the same notations as above, assume that

$$
\inf _{n \in \mathbb{Z}^{p}}|D(n)|>0
$$

Then $\mathrm{L}_{22}$ is invertible from $\mathrm{H}_{2}$ into itself and one has

$$
L_{22}^{-1} \varphi=\sum_{n \in \mathbb{Z}}-\frac{N_{22}(n)}{D(n)} c_{n}[\varphi] e^{2 i \pi n}, \forall \varphi \in H_{2}
$$

Remark 3.14. Note that (3.8) simply means that $\operatorname{det} A_{22} \neq 0$ and $D(n) \neq 0$ for all $n \in \mathbb{Z}^{p}$. The latter condition is not really restrictive if we play with the periodicity of the function space. Indeed, if we work on the space of $L=\left(L_{1}, \ldots, L_{p}\right)$-periodic function, the latter condition re-writes as $D(n / L) \neq 0$ for all $n \in \mathbb{Z}^{p}$ with $/ L=$ $\left(n_{1} / L_{1}, \ldots, n_{p} / L_{p}\right)$ so that one can find a suitable period vector $L$ such that this condition is satisfied as soon as $\operatorname{det} A_{22} \neq 0$ and $D(\xi)=0$ has a finite number of solutions.

Under the above assumption, namely (3.8), equation (2.5) in the periodic setting re-writes as the following ordinary differential equation in $H_{1}$

$$
\frac{\partial u(\cdot, t)}{\partial t}=\mathcal{A} u(\cdot, t) \text { with } \mathcal{A}=\left(L_{12}-L_{11} L_{22}^{-1} L_{21}\right) \in \mathcal{L}\left(H_{1}\right),
$$

that is, using Fourier coefficients as well as the above notations, equivalent to

$$
D(n) \frac{\mathrm{d}}{\mathrm{d} t} c_{n}[u(\cdot, t)]=M(n) c_{n}[u(\cdot, t)], \forall n \in \mathbb{Z}^{p} .
$$

Next we set

$$
P_{n}(z)=P(D(n) z ; M(n))=\operatorname{det}(D(n) z-M(n)), n \in \mathbb{Z}^{p}, z \in \mathbb{C} .
$$

And, in addition to (3.8), we assume that there exist $\alpha>0, \beta>0$ and $\varepsilon>0$ such that

$$
\inf _{n \in \mathbb{Z}^{p}, z \in S_{k}(\varepsilon)}\left|P_{n}(z)\right|>0, \forall k=-\alpha, \beta
$$

wherein we have set $S_{k}(\varepsilon)$ the complex strip given by

$$
S_{k}(\varepsilon)=\{z \in \mathbb{C}: k-\varepsilon \leq \operatorname{Re}(z) \leq k+\varepsilon\} .
$$

Under the above set of assumption it readily follows that the following splitting result holds.

Theorem 3.15 (Trichotomy splitting). The linear bounded operator $\mathcal{A} \in \mathcal{L}\left(H_{1}\right)$ is exponentially trichotomic in the sense that there exist three orthogonal projectors $\Pi^{k} \in \mathcal{L}\left(H_{1}\right)$ for $k=s, c$, u such that $\Pi^{s}+\Pi^{c}+\Pi^{u}=I$ and 
(i) one has $\Pi^{k} e^{t \mathcal{A}}=e^{t \mathcal{A}} \Pi^{k}$ for all $t \in \mathbb{R}$ and $k=s, c, u$;

(ii) there exist some constant $M>0$ and $\varepsilon_{0}>0$ such that

$$
\begin{aligned}
& \left\|e^{t \mathcal{A}} \Pi^{s}\right\| \leq M e^{-\alpha t}, \forall t \geq 0, \quad\left\|e^{-t \mathcal{A}} \Pi^{u}\right\| \leq M e^{-\beta t}, \forall t \geq 0, \\
& \left\|e^{t \mathcal{A}} \Pi^{c}\right\| \leq M e^{\left(\beta-\varepsilon_{0}\right) t}, \forall t \geq 0 \quad\left\|e^{-t \mathcal{A}} \Pi^{c}\right\| \leq M e^{\left(\alpha-\varepsilon_{0}\right) t}, \forall t \geq 0 .
\end{aligned}
$$

The proof of this result follows from a similar analysis than the one given above in the $L^{2}$-framework using Fourier coefficients.

Note that the condition (3.9) cannot hold true in the $L^{2}$-setting presented in the previous section since the spectrum takes the form of continuous curves. In the periodic setting the continuous curves is replaced by a countable sampling of the same curve so that this condition is reasonable.

Let us also mention that contrary to stable dichotomy - as obtained in the section above in the $L^{2}$-setting the exponential trichotomy is stable with respect to small perturbation (see for instance [20] and the references therein) and be used to construct invariant manifolds for nonlinear perturbation. This would also us to obtained results for the nonlinear problem such as problem (2.2). This will be studied in a forthcoming work.

\section{Application to Problem (2.4)}

We are now going to apply the theoretical results derived in the previous section to the special case of Problem (2.4). To that purpose let us observe that Problem (2.4) takes the form of (2.5) with $n_{1}=2$ and $n_{2}=1$, and it can be re-written as

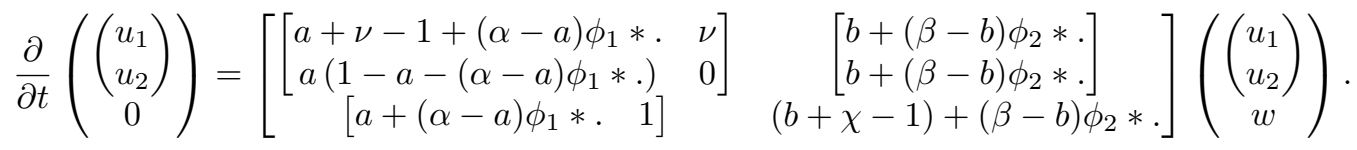

Here recall that the different parameters arising in this system of equations satisfy

$$
\nu>0, \chi \leq 0,0<a<\alpha<1,0<b<\beta, a+b=1 \text { and } \alpha+\beta>1 .
$$

For this system, only initial data the initial condition $u_{1}(\cdot, 0)=u_{1}^{0} \in L^{2}\left(\mathbb{R}^{p}, \mathbb{R}\right)$ is given and fixed and the problem is supplemented together with the weak transversality condition

$$
\lim _{t \rightarrow \infty}\left(u_{1}, u_{2}\right)^{T}(\cdot, t)=0 \text { for the topology of } L^{2}\left(\mathbb{R}^{p}, \mathbb{R}^{2}\right) .
$$

In the above system of equations, we have assumed without loose of generality that the parameter $d$ arising in (2.4) satisfies $d=1$. Note that such a simplification simply follows from a time rescaling argument.

The goal of this section consists in determining whether the initial condition $u_{2}(\cdot, 0)=u_{2}^{0} \in L^{2}\left(\mathbb{R}^{p} ; \mathbb{R}\right)$ exists and could be uniquely defined in order that the dynamical system (4.1) admits a forward solution. This analysis is based on the dichotomy property stated in Theorem 3.7.

Considering (4.1) we assume that the kernels $\phi_{1}$ and $\phi_{2}$ both belong to $L_{+}^{1}\left(\mathbb{R}^{p}, \mathbb{R}\right)$ and they furthermore satisfy the symmetry and the unit mass condition

$$
\phi_{2}(-x)=\phi_{2}(x) \text { a.e. } x \in \mathbb{R}^{p} \text { and } \int_{\mathbb{R}^{p}} \phi_{i}(x) \mathrm{d} x=1, \forall i=1,2 .
$$

In that context, the functions and matrix valued functions arising in the Assumptions 3.4 and 3.6, in the previous section, read as follows:

$$
D(\xi)=(b+\chi-1)+(\beta-b) \widehat{\phi_{2}}(\xi),
$$


and, by setting

$$
\pi(\xi):=\left((\alpha-a) \widehat{\phi_{1}}(\xi)+a-1\right)(\chi-1) \text { and } \mu(\xi)=(\beta-b) \widehat{\phi_{2}}(\xi)+b
$$

the matrix $M \equiv M(\xi)$ takes the form

$$
M(\xi)=\left[\begin{array}{cc}
\pi(\xi)-\mu(\xi)+\nu D(\xi) & \nu D(\xi)-\mu(\xi) \\
a[\mu(\xi)-\pi(\xi)] & a \mu(\xi)
\end{array}\right]
$$

while the matrix $M^{\infty}$ is defined by

$$
M^{\infty}:=\lim _{\|\xi\| \rightarrow \infty} M(\xi)=\left[\begin{array}{cc}
(a-1)(\chi-1)-b+\nu(b+\chi-1) & \nu(b+\chi-1)-b \\
a[b-(a-1)(\chi-1)] & a b
\end{array}\right] .
$$

Note that, due to (4.2), one has

$$
\lim _{\|\xi\| \rightarrow \infty} D(\xi)=b+\chi-1<0 \text { and } \operatorname{det} M^{\infty}=\nu a(1-a)(1-\chi)[b+\chi-1]<0,
$$

so that the condition on the real matrix $M^{\infty}$ arising in Assumption 3.6 is satisfied.

Before going further, let us observe that the symmetry property in (4.3) ensures that the function $D$ has real values. In the sequel we assume that it satisfies

$$
\left\{\xi \in \mathbb{R}^{p}: D(\xi)=0\right\} \text { is a finite set. }
$$

We are now interesting in locating the eigenvalues of the matrix $M(\xi)$. To that aim one may observe that the characteristic polynomial of matrix $M(\xi)$ reads as

$$
P(z, M(\xi))=z^{2}-\operatorname{Tr}(\xi) z+\nu a \pi(\xi) D(\xi), \quad z \in \mathbb{C}, \xi \in \mathbb{R}^{p},
$$

where $\operatorname{Tr}(\xi)$ denotes the trace of the matrix $M(\xi)$, that reads as follows:

$$
\operatorname{Tr}(\xi)=\pi(\xi)+\nu D(\xi)+(a-1) \mu(\xi) .
$$

We shall now split our study into two parts. We firstly deal with the case where the kernel $\phi_{1}$ is symmetric and then we turn to the case where $\phi_{2}$ is not symmetric, so that its Fourier transform may take complex but non real values.

\subsection{Case of symmetric kernel $\phi_{1}$}

In this section, in addition to (4.3), (4.8) and (4.9), we assume that the kernel $\phi_{1}$ is symmetric, that reads as $\phi_{1}(-x)=\phi_{1}(x)$ for almost every $x \in \mathbb{R}^{p}$. In that framework, the matrix $M(\xi)$ defined above in (4.6) has real entries.

Now, by recalling (4.2) and (4.3), let us observe that

$$
\pi(\xi)>0, \forall \xi \in \mathbb{R}^{p} .
$$

Now in order to re-write Assumption 3.6 in the context of this application we assume that

$$
\begin{aligned}
& \left\{\xi \in \mathbb{R}^{p}: \operatorname{Tr}(\xi)=0 \text { and } D(\xi) \geq 0\right\}=\left\{\xi_{1}, \ldots, \xi_{l}\right\} \\
& D\left(\xi_{j}\right) \neq 0, \forall j=1, \ldots, l
\end{aligned}
$$


Using the above assumption and recalling (4.8) and (4.9), it is easy to check that Assumption 3.6.

Now let us denote by $\Lambda^{+}$(resp. $\left.\Lambda^{-}\right)$the root of $P(z, M(\xi))=0$ with greatest (resp. smallest) real part. Next let us introduce the three open sets

$$
\begin{aligned}
G & =\left\{\xi \in \mathbb{R}^{p}: \operatorname{Re} \Lambda^{-}(\xi)<0<\operatorname{Re} \Lambda^{+}(\xi)\right\}=\left\{\xi \in \mathbb{R}^{p}: D(\xi)<0\right\}, \\
F^{s} & =\left\{\xi \in \mathbb{R}^{p}: \operatorname{Re} \Lambda^{-}(\xi) \leq \operatorname{Re} \Lambda^{+}(\xi)<0\right\}=\left\{\xi \in \mathbb{R}^{p}: D(\xi)>0 \text { and } \operatorname{Tr}(\xi)<0\right\}, \\
F^{u} & =\left\{\xi \in \mathbb{R}^{p}: 0<\operatorname{Re} \Lambda^{-}(\xi) \leq \operatorname{Re} \Lambda^{+}(\xi)\right\}=\left\{\xi \in \mathbb{R}^{p}: D(\xi)>0 \text { and } \operatorname{Tr}(\xi)>0\right\} .
\end{aligned}
$$

Note that, recalling (4.12), one gets the following disjoint decomposition of $\mathbb{R}^{p}$ :

$$
\mathbb{R}^{p}=G \cup F^{s} \cup F^{u} \cup\left\{\xi_{1}, \ldots, \xi_{l}\right\} \cup\left(D^{-1}\{0\}\right) .
$$

Note that $\left\{\xi_{1}, \ldots, \xi_{l}\right\}$ provides the set of parameters ensuring the existence of a pair of non zero purely imaginary eigenvalues for $M(\xi)$ while $D^{-1}(\{0\})$ provides the set of parameters $\xi$ for which the matrix $M(\xi)$ has the (simple) zero eigenvalue. Let us also note that for $\xi \in G$ then the two eigenvalues $\Lambda^{+}(\xi)$ and $\Lambda^{-}(\xi)$ are both real. Moreover note that for each $\xi \in G \cup F^{s} \cup F^{u}$, the spectral projectors associated to $M(\xi)$ satisfy the following properties

$$
\begin{aligned}
& \Pi^{c u}(\xi)=\Pi^{u}(\xi) \text { and } \Pi^{c s}(\xi)=\Pi^{s}(\xi), \forall \xi \in G, \text { and they are one-dimensional, } \\
& \Pi^{c s}(\xi)=I_{2}, \forall \xi \in F^{s} \text { and } \Pi^{c u}(\xi)=I_{2}, \forall \xi \in F^{u} \text {. }
\end{aligned}
$$

We are able to state the main result of this section.

Theorem 4.1. Let the initial data $u_{1}^{0} \in L^{2}\left(\mathbb{R}^{p} ; \mathbb{R}\right)$ be given and fixed. Under the above set of assumptions, and assume furthermore that

$$
\inf _{\xi \in G}|\nu D(\xi)-\mu(\xi)|>0
$$

then the following hold true:

(a) If $G=\mathbb{R}^{p}$, there exists a unique $u_{2}^{0} \in L^{2}\left(\mathbb{R}^{p} ; \mathbb{R}\right)$ such that System (4.1) has a 2 -integrated forward solution $\left(u_{1}, u_{2}, w\right)$ (in the sense of Def. 2.1) when equipped with the initial condition $\left(u_{1}^{0}, u_{2}^{0}\right)^{T} \in L^{2}\left(\mathbb{R} ; \mathbb{R}^{2}\right)$ and satisfying the weak transversality condition

$$
\left(u_{1}(\cdot, t), u_{2}(\cdot, t)\right) \rightarrow(0,0) \text { in } L^{2}\left(\mathbb{R}^{p} ; \mathbb{R}^{2}\right) \text { as } t \rightarrow \infty
$$

In that case the very weak solution are 2-integrated solutions in the sense of Definition 2.1. This situation will be referred as local existence and uniqueness of the solution;

(b) Assume that $F^{s} \neq \emptyset$ and $u_{1}^{0} \in L^{2}\left(\mathbb{R}^{p} ; \mathbb{R}\right)$ satisfies $\widehat{u}_{1}^{0}(\xi)=0$ for almost any $\xi \in F^{u}$. Then there exists a family $\mathcal{I}$ of functions $u_{2}^{0} \in L^{2}\left(\mathbb{R}^{p} ; \mathbb{R}\right)$, such that for each initial data $\left(u_{1}^{0}, u_{2}^{0}\right)$ with $u_{2}^{0} \in \mathcal{I}$, System (4.1) has a very weak forward solution (in the sense of Def. 3.1) that satisfies the weak transversality condition as above.

In this case we will say that there is local indeterminacy while the measure meas $\left(F^{s}\right)$ will be refereed as the degree of indeterminacy

Remark 4.2. If $F^{u} \neq \emptyset$ then $(b)$ holds true for (very weak) backward solutions. In that case one may speak about local backward indeterminacy.

Proof. The proof of the above result relies on Theorem 3.7, Theorem 3.8 and on the representation of the projector $\mathcal{P}^{s}$ in term of the spectral projectors $\Pi^{\alpha}(\xi)$, with $\alpha=c s, c u$ associated to the matrix $M(\xi)$. Recalling 
(4.13), note that the projector $\mathcal{P}^{s}$ on $L^{2}\left(\mathbb{R}^{p} ; \mathbb{R}^{2}\right)$ is given, for any $\varphi \in L^{2}\left(\mathbb{R}^{p} ; \mathbb{R}^{2}\right)$, by

$$
\widehat{\left(\mathcal{P}^{s} \varphi\right)}(\xi)=\left[\chi_{G}(\xi)\left[\Pi^{u}(\xi) \chi_{\{D(\xi)<0\}}\right]+\chi_{F^{s}}(\xi) \Pi^{c s}(\xi)\right] \widehat{\varphi}(\xi) .
$$

We are now able to prove (a). To that aim let us first observe that, since $G=\mathbb{R}^{p}$ then $D(\xi)<0$ for all $\xi \in \mathbb{R}^{p}$ so that the assumptions of Theorem 3.8 are satisfied. Next observe that the condition

$$
\left(I-\mathcal{P}^{s}\right)\left(\begin{array}{l}
u_{1}^{0} \\
u_{2}^{0}
\end{array}\right)=0
$$

re-writes as

$$
\left(\begin{array}{l}
\widehat{u}_{1}^{0}(\xi) \\
\widehat{u}_{2}^{0}(\xi)
\end{array}\right)=\Pi^{u}(\xi)\left(\begin{array}{c}
\widehat{u}_{1}^{0}(\xi) \\
\widehat{u}_{2}^{0}(\xi)
\end{array}\right), \xi \in \mathbb{R}^{p} \Leftrightarrow \Pi^{s}(\xi)\left(\begin{array}{c}
\widehat{u}_{1}^{0}(\xi) \\
\widehat{u}_{2}^{0}(\xi)
\end{array}\right)=\left(\begin{array}{l}
0 \\
0
\end{array}\right), \xi \in \mathbb{R}^{p}
$$

This re-writes as

$$
\widehat{u}_{2}^{0}(\xi)=\frac{a \mu(\xi)-\Lambda^{-}(\xi)}{\nu D(\xi)-\mu(\xi)} \widehat{u}_{1}^{0}(\xi)
$$

Since the right hand side of this inequality denotes a function in $L^{2}\left(\mathbb{R}^{p} ; \mathbb{C}\right)$ and since the function $\xi \mapsto \frac{a \mu(\xi)-\Lambda^{-}(\xi)}{\nu D(\xi)-\mu(\xi)}$ has real values, this completes the proof of $(a)$ by applying the inverse Fourier transform.

Now in order to prove $(b)$ let us observe that the condition

$$
\left(I-\mathcal{P}^{s}\right)\left(\begin{array}{l}
u_{1}^{0} \\
u_{2}^{0}
\end{array}\right)=0
$$

re-writes for almost every $\xi \in \mathbb{R}^{p}$ as

$$
\left\{\begin{array}{l}
\widehat{u}_{1}^{0}(\xi) \chi_{\mathbb{R}^{p} \backslash G}(\xi)=\widehat{u}_{1}^{0}(\xi) \chi_{F^{s}}(\xi) \\
\widehat{u}_{2}^{0}(\xi)=\frac{a \mu(\xi)-\Lambda^{-}(\xi)}{\nu D(\xi)-\mu(\xi)} \chi_{G}(\xi) \widehat{u}_{1}^{0}(\xi)+\chi_{F^{s}}(\xi) \widehat{u}_{2}^{0}(\xi)
\end{array}\right.
$$

Hence if we denote by $\mathcal{I}$ the set of functions defined by

$$
\mathcal{I}=\left\{u_{2}^{0} \in L^{2}\left(\mathbb{R}^{p} ; \mathbb{R}\right): \widehat{u}_{2}^{0}(\xi)=\frac{a \mu(\xi)-\Lambda^{-}(\xi)}{\nu D(\xi)-\mu(\xi)} \widehat{u}_{1}^{0}(\xi), \text { a.e. } \xi \in G \text { and } \widehat{u}_{2}^{0}(\xi)=0, \text { a.e. } \xi \in F^{u}\right\}
$$

then (b) follows by applying Theorem 3.7. This completes the proof of Theorem 4.1.

We are not only interested in determining the set of parameters for which indeterminacy exists, but also in computing the size of this indeterminacy. Polemarchakis [35] proposes, for finite dimensional systems such as ordinary differential equations or map, to define the degree of indeterminacy as an integer $K \in \mathbb{N}$, such as the dynamical system which is considered, admits a family of solutions parametrized by $K$-dimensional parameters $\alpha \in \mathbb{R}^{K}$. This definition is based on the comparison between the number of eigenvalues with positive real part and the number of missing initial conditions [14]. This can also be applied to infinite dimensional framework as for delay differential equations [2].

Theorem 4.1 enables us to, in some sense, extend the definition of degree of indeterminacy to spatially distributed systems as the ones we consider in this work. Our definition reads as follows 
Definition 4.3. If $F^{u}=\emptyset$, then the degree of indetermination is the measure of the set $F^{s}$. It measures the size of the frequencies leading to local indeterminacies.

Indeed, if $F^{u}=\emptyset$, according to Theorem 4.1, a solution of the spatially structured system is characterized by an initial condition $\left(u_{1}^{0}, u_{2}^{0}\right)^{T}$ with $u_{2}^{0} \in \mathcal{I}$ defined above in the proof of Theorem 4.1. Hence frequencies in $F^{s}$ are not fully determined and indeterminacy is thus parametrized by $K \in \mathbb{R}$ such that $K$ is equal to the meas $\left(F^{s}\right)$ missing frequencies of $\widehat{u}_{2}^{0}$. In our problem, these frequencies correspond to small frequencies since that high frequencies belong to $G$.

Moreover, if we consider two functions $u_{21}$ and $u_{22}$ in $\mathcal{I}$, then one has

$$
u_{21}(x)-u_{22}(x)=\mathcal{F}^{-1}\left[\xi \mapsto \chi_{F^{s}}(\xi)\left(\widehat{u}_{21}(\xi)-\widehat{u}_{22}(\xi)\right)\right](x), \text { a.e. } x \in \mathbb{R}^{p}
$$

Hence since meas $\left(F^{s}\right)<\infty$, the non-knowledge of the initial data $\widehat{u}_{2}^{0}(\xi)$ for all $\xi \in F^{s}$ impacts $u_{2}^{0}$ in a non local way, and more specifically on the whole space $x \in \mathbb{R}^{p}$.

Now in view of Theorem 4.1 we provide simple conditions on the parameters of Problem (4.1) leading to local uniqueness and local indeterminacies.

Lemma 4.4. Under the same assumptions that the ones of Theorem 4.1 the following hold true:

(i) If $\beta+\chi-1<0$ then $G=\mathbb{R}^{p}$ and local existence and uniqueness occurs.

(ii) If $\beta+\chi-1>0$ then $F^{s} \cup F^{u} \neq \emptyset$ and local indeterminacy occur (for backward or forward solutions).

Remark 4.5. Note that the condition $\beta+\chi-1<0$ is the condition for the ODE problem (without spatial interaction) to be determinate (see [4]). Therefore in that case, no indeterminacy appears due to spatial interactions.

Proof. To prove (i) let us observe that one has

$$
D(\xi) \leq(\beta-b)+b+\chi-1<0, \forall \xi \in \mathbb{R}^{p} .
$$

This property re-writes as $G=\mathbb{R}^{p}$.

The second point, namely $(i i)$ directly follows from $D(0)=\beta+\chi-1>0$ together with (4.12).

The spectral situation corresponding to $(i)$ in the above lemma is described in Figure 1 while the second point (ii) may correspond to more complicated spectral branches. Some of these configurations are described in Figure 2.

In the rest of this section we give two examples of spatial interactions and we only focus on the one-dimensional case, that is $p=1$. To that aim we deal with the case of Gaussian kernels.

Corollary 4.6 (Gaussian kernel). If $\phi_{1}$ is symmetric and $\phi_{2}$ is a symmetric Gaussian kernel with standard deviation parameter $\sigma$, then the degree of indetermination is decreasing with respect to $\sigma$ for forward solutions in the case where $\nu \leq 1-a$ and for backward solution else.

Proof. We first notice as $\phi_{2}$ is a symmetric Gaussian function with variance $\sigma>0$, one has

$$
\widehat{\phi_{2}}(\xi)=e^{-\pi^{2} \sigma^{2} \xi^{2}}
$$

We explicitly write down the dependence with respect to $\sigma$ by writing $f(\xi ; \sigma)$ instead of $f(\xi)$ for functions depending on $\xi$. For instance we write $D(\xi ; \sigma)$ and $\operatorname{Tr}(\xi ; \sigma)$ instead of $D(\xi)$ and $\operatorname{Tr}(\xi)$ respectively (see $(4.4)$ and (4.11)). First note that for all $\xi \in \mathbb{R}$ and $0<\sigma_{1}<\sigma_{2}$ one has

$$
D\left(\xi ; \sigma_{1}\right) \geq D\left(\xi ; \sigma_{2}\right) \text { and }
$$




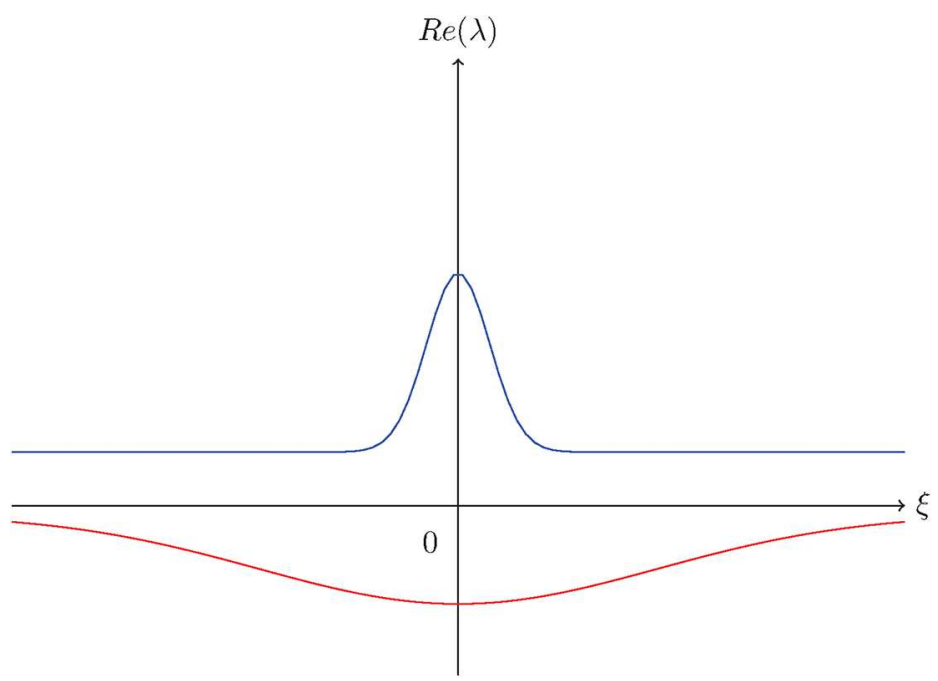

Figure 1. Real part of the two eigenvalues $\Lambda^{ \pm}(\xi)$ for the case $\beta+\chi-1<0$ and $p=1$.

Next if $\nu \leq 1-a($ resp. $\nu>1-a)$ then one gets for all $\xi \in \mathbb{R}$ and $0<\sigma_{1}<\sigma_{2}$ that

$$
\operatorname{Tr}\left(\xi ; \sigma_{1}\right) \leq \operatorname{Tr}\left(\xi, \sigma_{2}\right) \quad\left(\text { resp. } \operatorname{Tr}\left(\xi ; \sigma_{1}\right)>\operatorname{Tr}\left(\xi, \sigma_{2}\right)\right) .
$$

As a consequence the corresponding set $F^{s}\left(\sigma_{1}\right)$ and $F^{s}\left(\sigma_{2}\right)\left(\right.$ resp. $F^{u}\left(\sigma_{1}\right)$ and $\left.F^{u}\left(\sigma_{2}\right)\right)$ satisfy $F^{s}\left(\sigma_{2}\right) \subset F^{s}\left(\sigma_{1}\right)$ (resp. $\left.F^{u}\left(\sigma_{2}\right) \subset F^{u}\left(\sigma_{1}\right)\right)$ and the statement follows.

\subsection{Case of asymmetric kernel $\phi_{1}$}

The previous subsection only deals with the case of symmetric interactions among neighbours. However, asymmetric conditions can also arise in structured systems and in particular in the economic system we consider in this work. Let us assume in this paragraph that $\phi_{1}$, which models the weight of neighbours capital, is asymmetric while $\phi_{2}$ is symmetric (see (4.3)). As far as an asymmetric kernel $\phi_{1}$ is considered, the function $\xi \mapsto \widehat{\phi_{1}}(\xi)$ takes complex (non real values).

In that case the characteristic polynomial of matrix $M(\xi)$ can be written, for any $z \in \mathbb{C}$ and $\xi \in \mathbb{R}^{p}$, as

$$
P(z, M(\xi))=z^{2}-(\operatorname{Re}(\operatorname{Tr}(\xi))+i \operatorname{Im}(\operatorname{Tr}(\xi))) z+\nu a D(\xi)(\operatorname{Re}(\pi(\xi))+i \operatorname{Im}(\pi(\xi))),
$$

where (see (4.4) and (4.5))

$$
\begin{aligned}
\operatorname{Re}(\operatorname{Tr}(\xi))= & (\chi-1)\left[(\alpha-a) \operatorname{Re}\left(\widehat{\phi_{1}}(\xi)\right)+\nu+a-1\right] \\
& +(\nu+a-1)\left[(\beta-b) \widehat{\phi_{2}}(\xi)+b\right] \\
\operatorname{Im}(\operatorname{Tr}(\xi))= & (\chi-1)(\alpha-a) \operatorname{Im}\left(\widehat{\phi_{1}}(\xi)\right) \\
\operatorname{Re}(\pi(\xi))= & (\chi-1)\left[(\alpha-a) \operatorname{Re}\left(\widehat{\phi_{1}}(\xi)\right)+a-1\right] \\
\operatorname{Im}(\pi(\xi))= & \operatorname{Im}(\operatorname{Tr}(\xi))
\end{aligned}
$$




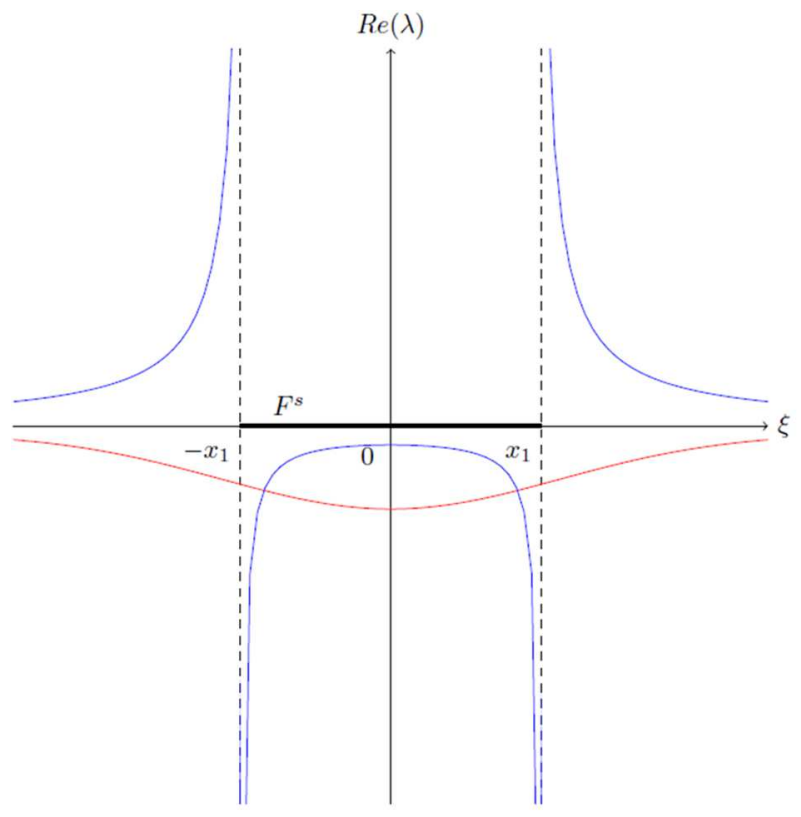

(a)

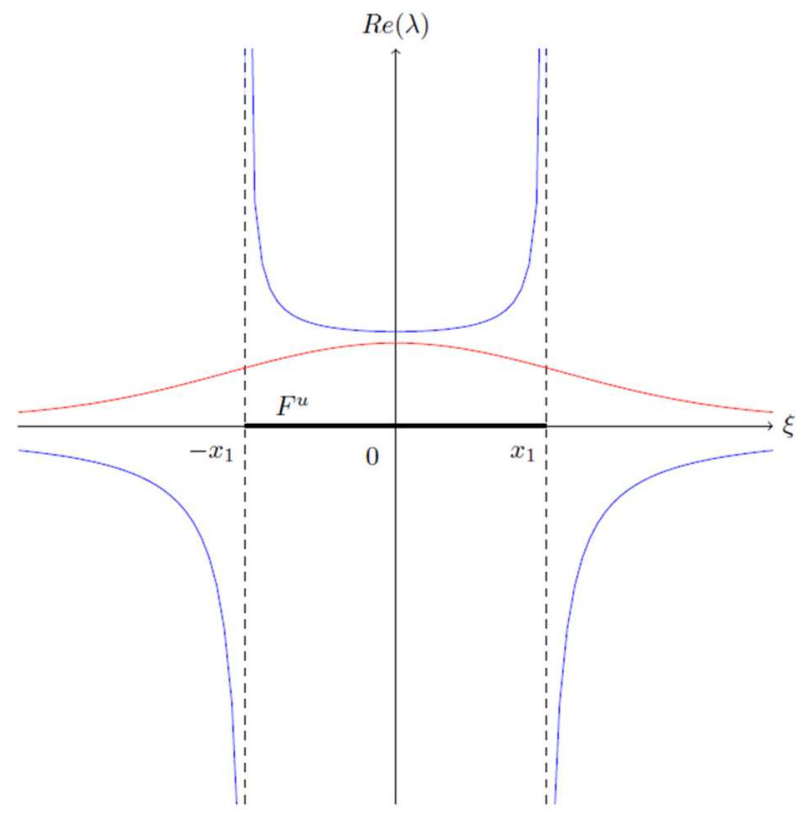

(b)

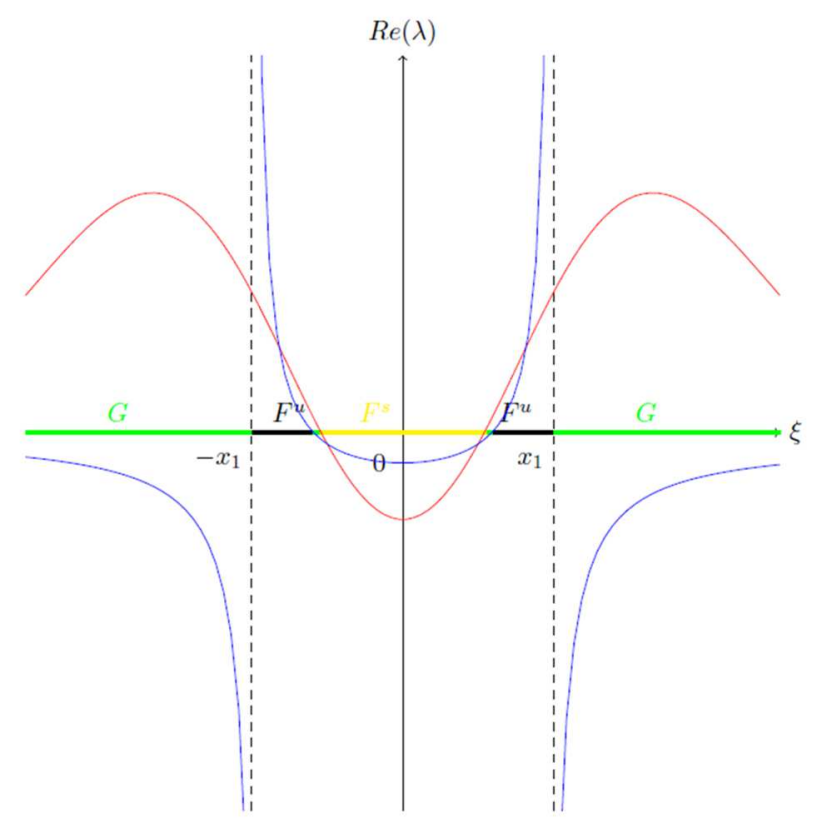

(c)

Figure 2. Real part of the two eigenvalues $\Lambda^{ \pm}(\xi)$ (red and blue lines) for the case $\beta+\chi-1>0$ and $p=1$. 
Let us first notice (see Appendix A) that the equation $P(z, M(\xi))=0$ has a purely imaginary root if and only if $\xi \in \mathcal{R}$ where the set $\mathcal{R}$ is defined as follows:

$$
\mathcal{R}=\left\{\xi \in \mathbb{R}^{p}: \operatorname{Im}(\operatorname{Tr}(\xi))^{2}+4 \nu a D(\xi) \operatorname{Re} \pi(\xi) \geq 0 \text { and }\left\{\begin{array}{l}
\operatorname{Re} \operatorname{Tr}(\xi)=0 \text { and } D(\xi) \operatorname{Im} \pi(\xi)=0 \\
\text { or } \\
\operatorname{Tr}(\xi) \neq 0 \text { and } D(\xi) \Psi(\xi)=0
\end{array}\right\}\right.
$$

wherein we have set for each $\xi \in \mathbb{R}^{p}$ such that $\operatorname{Tr}(\xi) \neq 0$ :

$$
\Psi(\xi)=\operatorname{Re}(\pi(\xi))-\left(\frac{\operatorname{Im}(\pi(\xi))}{\operatorname{Re}(\operatorname{Tr}(\xi))}\right)^{2}(\nu a D(\xi)-\operatorname{Re}(\operatorname{Tr}(\xi))) .
$$

In order to satisfy Assumption 3.6 we assume that the set $\mathcal{R}$ is finite and we also assume that the corresponding imaginary roots are simple.

As it has been seen before the sets $G, F^{s}$ and $F^{u}$ defined in the left-hand side of (4.13) play an important role in the description of indeterminacies. Within this asymmetric framework the same analysis as above can be performed and, here again the existence and the description of indeterminacies follow from the description of the sets $G, F^{s}$ and $F^{u}$. Since Theorem 4.1 can be easily extended to this asymmetric context, we only focus in this part on the description of the above mentioned sets in term of $\operatorname{Tr}(\xi), \pi(\xi)$ and $D(\xi)$. This is fulfilled using Talbot lemma for the localization of the roots of a complex polynomial of degree two (see Appendix A)

Lemma 4.7. Then the sets $G, F^{s}, F^{u}$ are characterized as follows

$$
\begin{aligned}
G & =\left\{\xi \in \mathbb{R}^{p} \backslash \mathcal{R}:\left\{\begin{array}{c}
\operatorname{Re}(\operatorname{Tr}(\xi))=0 \\
\text { or } \\
\operatorname{Re}(\operatorname{Tr}(\xi))>0 \text { and } D(\xi) \Psi(\xi)<0 \\
\text { or } \\
\operatorname{Re}(\operatorname{Tr}(\xi))<0 \text { and } D(\xi) \Psi(\xi)>0
\end{array}\right\},\right. \\
F^{s} & =\left\{\xi \in \mathbb{R}^{p} \backslash \mathcal{R}: \operatorname{Re}(\operatorname{Tr}(\xi))<0 \text { and } D(\xi) \Psi(\xi)>0\right\} \\
F^{u} & =\left\{\xi \in \mathbb{R}^{p} \backslash \mathcal{R}: \operatorname{Re}(\operatorname{Tr}(\xi))>0 \text { and } D(\xi) \Psi(\xi)>0\right\}
\end{aligned}
$$

Proof. Let us first observe that when $\operatorname{Re}(\operatorname{Tr}(\xi))=0$ then $P(z, M(\xi))$ has two roots with opposite real parts. Therefore such a situation belongs to the set $G$ expect if the two roots belongs to the imaginary axis. However this latter configuration means that $\xi \in \mathcal{R}$.

From now one we assume that $\operatorname{Re}(\operatorname{Tr}(\xi)) \neq 0$ and $\xi \in \mathbb{R}^{p} \backslash \mathcal{R}$. In the sequel, for each polynomial $P \in \mathbb{C}[X]$ we denote by $r(P)$ the number of roots with non negative real part. Hence the characteristic equation $P(z, M(\xi))$ has two roots with negative real part means that $r(P(z, M(\xi)))=0$. Using Talbot lemma it thus implies that

$$
\begin{aligned}
& 2=\operatorname{sign}\left(-\frac{1}{\operatorname{Re}(\operatorname{Tr}(\xi))}\right)+\operatorname{sign}\left(\frac{-\operatorname{Re}(\operatorname{Tr}(\xi))}{\nu a D(\xi) \operatorname{Re}(\pi(\xi))-\frac{\nu a D(\xi) \operatorname{Im}(\pi(\xi))}{\operatorname{Re}(\operatorname{Tr}(\xi))}\left(\frac{\nu a D(\xi) \operatorname{Im}(\pi(\xi))}{\operatorname{Re}(\operatorname{Tr}(\xi))}-\operatorname{Im}(\operatorname{Tr}(\xi))\right)}\right), \\
& 2=\operatorname{sign}\left(-\frac{1}{\operatorname{Re}(\operatorname{Tr}(\xi))}\right)+\operatorname{sign}\left(\frac{-\operatorname{Re}(\operatorname{Tr}(\xi))}{\nu a D(\xi)\left[\operatorname{Re}(\pi(\xi))-\left(\frac{\operatorname{Im}(\pi(\xi))}{\operatorname{Re}(\operatorname{Tr}(\xi))}\right)^{2}(\nu a D(\xi)-\operatorname{Re}(\operatorname{Tr}(\xi)))\right]}\right),
\end{aligned}
$$

which is satisfied if and only if $\operatorname{Re}(\operatorname{Tr}(\xi))<0$ and $D(\xi) \Psi(\xi)>0$. 
Next the characteristic equation has two roots with positive real part is $r(P(z, M(\xi)))=2$. It thus implies that

$$
-2=\operatorname{sign}\left(-\frac{1}{\operatorname{Re}(\operatorname{Tr}(\xi))}\right)+\operatorname{sign}\left(\frac{-\operatorname{Re}(\operatorname{Tr}(\xi))}{\nu a D(\xi)\left[\operatorname{Re}(\pi(\xi))-\left(\frac{\operatorname{Im}(\pi(\xi))}{\operatorname{Re}(\operatorname{Tr}(\xi))}\right)^{2}(\nu a D(\xi)-\operatorname{Re}(\operatorname{Tr}(\xi)))\right]}\right),
$$

which is satisfied if and only if $\operatorname{Re}(\operatorname{Tr}(\xi))>0$ and $D(\xi) \Psi(\xi)>0$.

Finally the characteristic equation has exactly one root with positive real part means that $r(P(z, M(\xi)))=1$. This implies

$$
0=\operatorname{sign}\left(-\frac{1}{\operatorname{Re}(\operatorname{Tr}(\xi))}\right)+\operatorname{sign}\left(\frac{-\operatorname{Re}(\operatorname{Tr}(\xi))}{\nu a D(\xi)\left[\operatorname{Re}(\pi(\xi))-\left(\frac{\operatorname{Im}(\pi(\xi))}{\operatorname{Re}(\operatorname{Tr}(\xi))}\right)^{2}(\nu a D(\xi)-\operatorname{Re}(\operatorname{Tr}(\xi)))\right]}\right),
$$

and this condition is satisfied if and only if $\left\{\begin{array}{c}\operatorname{Re}(\operatorname{Tr}(\xi))>0 \text { and } D(\xi) \Psi(\xi)<0 \\ \text { or }(\operatorname{Tr}(\xi))<0 \text { and } D(\xi) \Psi(\xi)>0\end{array}\right.$.

We complete this section by proving that indeterminacies may be displayed even in the case where the determinate condition $\beta+\chi-1<0$ is satisfied. Recall that under the condition on the parameter, the ODE system without spatial interaction is determinate as well as the spatial structured problem with symmetric interactions (see Lem. 4.4 and Rem. 4.5). However when asymmetric spatial interactions are considered then we will show below that indeterminacies may occur under the determinate condition $\beta+\chi-1<0$.

To see this we fix any symmetric kernel $\phi_{2}$ and we choose a kernel $\phi_{1}$ such that

$$
\widehat{\phi_{1}}(\xi)=g(\xi) e^{i m \xi}
$$

for some $m>0$ and where $g(\mathbb{R}) \subset \mathbb{R}$. Note that such a choice can be obtained from any symmetric kernel $\varphi$ by considering the shifted kernel $\phi_{1}(x)=\varphi\left(x-\frac{m}{2 \pi}\right)$.

Next observe that $\widehat{\phi_{1}}(0)=1$ implies that $g(0)=1$. In the sequel we shall find a suitable parameter set satisfying (4.2), the 'determinate' $\beta+\chi-1<0$ condition as well as the indeterminacy criterion $F^{u} \cup F^{s} \neq \emptyset$. To that aim recall that, since $\beta+\chi-1<0$, one has $D(\xi)<0$ for $\xi \in \mathbb{R}^{p}$. Moreover observe that

$$
\Psi\left(\frac{\xi}{m}\right) \rightarrow \Psi^{\infty}(\xi)
$$

locally uniformly with respect to $\xi$ as $m \rightarrow \infty$ and where $\psi^{\infty}(\xi)$ is the function defined by

$$
\begin{aligned}
& \Psi^{\infty}(\xi)=(\chi-1)[(\alpha-a) \cos \xi+a-1] \\
& -\left(\frac{(\chi-1)(\alpha-a) \sin \xi}{(\chi-1)(\alpha-a) \cos \xi+(\nu+a-1)(\beta+\chi-1)}\right)^{2}[\nu a \beta+(\chi-1)(\nu-1)(a-1)-(\chi-1)(\alpha-a) \cos \xi] .
\end{aligned}
$$

We now choose $\xi=\frac{\pi}{2}$ and we claim that we can find $0<a<<1$ and $\nu>0$ such that $\Psi^{\infty}\left(\frac{\pi}{2}\right)<0$. It is easy to observe that the proof of such a claim is sufficient to conclude that the above statement holds true. 
To prove this claim note that one has

$$
\begin{aligned}
\Psi^{\infty}\left(\frac{\pi}{2}\right)= & (\chi-1)[a-1] \\
& -\left(\frac{(\chi-1)(\alpha-a)}{(\nu+a-1)(\beta+\chi-1)}\right)^{2}[\nu a \beta+(\chi-1)(\nu-1)(a-1)] .
\end{aligned}
$$

We write down the dependence of the above expression with respect to $a, \nu$ and $\chi$, as $F(a, \nu, \chi)$. Hence one gets, by choosing $\nu=\nu_{\alpha}:=1+\frac{\alpha^{2}}{2}$

$$
\lim _{\chi \rightarrow-\infty, a \rightarrow 0} \frac{1}{\chi} F\left(a, \nu_{\alpha}, \chi\right)=-1+\frac{\alpha^{2}}{\nu_{\alpha}-1}=1 .
$$

This means that for $\chi<0$ and large enough and $a>0$ small enough, one has $\Psi^{\infty}\left(\frac{\pi}{2}\right)<0$ by choosing $\nu=\nu_{\alpha}$.

This computation shows that indeterminacy can occur, as far as an asymmetric kernel $\phi_{1}$ is considered, in the case $\beta-1+\chi<0$. On the contrary to the situation described by Benhabib and Farmer [4], indeterminacy can happen in the classical case in which wage is obtained as the intersection of an upward labour supply curve (which slope is $-\chi$ ) and of a labour demand curve with negative slope $\beta-1$, for $\beta<1$.

\section{CONCLUSiOn}

We provide a methodology to characterize the conditions for existence and uniqueness of a solution for a general dimension system of non local algebraic-differential equations, where space variables belong to $\mathbb{R}^{p}$ for some $p \geq 1$. Our method is based on the Fourier Transform of the problem and on the computation of the projector on the stable manifold. As the spectrum is continuous, properties of existence and uniqueness are obtained by characterizing the size of the set of Fourier parameters for which the characteristic roots have both positive real part. Under some conditions, we prove that a spectral dichotomy of the space of initial conditions can be obtained. This dichotomy enables the set of initial conditions to be split into those leading to (weak) solutions that converge to the steady state and those that do not. Using projection on the unstable manifold we manage to characterize the set of initial conditions that yields to an equilibrium.

We illustrate our methodology with a simple example of a growth model satisfying spatial production externalities. For the model being considered, we have proved that the neighborhood interaction kernels play an important role in determining whether or not the solution is unique. We have shown that the spatial interactions we considered do not increase the set of parameters that generate indeterminacy.

We have also shown that the degree of indeterminacy greatly depends on the properties of the interaction kernel, and, in general, is difficult to characterize. However, it has been seen that for Gaussian kernels, the higher the standard deviation parameter, the lower the degree of indeterminacy. Moreover, we have proved that for some asymmetric kernels, indeterminacy can occur for labor supply and demand curves with standard slopes.

\section{Appendix A}

\section{A.1 Coppel's lemma}

This paragraph recalls some exponential matrix estimates provided by Coppel (see [17], Prop. 3)

Lemma A.1. Let $A$ be an $n \times n$ matrix (for some $n \geq 1$ ) such that $s(A) \leq \alpha$ and $\|A\| \leq M$ for some given constant $\alpha \in \mathbb{R}$ and $M>0$. Then one has

$$
\left\|e^{t A}\right\| \leq e^{\alpha t} \sum_{k=0}^{n-1} \frac{(2 M t)^{k}}{k !}, \forall t \geq 0,
$$


and for each $\varepsilon \in(0,2 M)$ one has

$$
\left\|e^{t A}\right\| \leq\left(\frac{2 M}{\varepsilon}\right)^{n-1} e^{(\alpha+\varepsilon) t}, \quad \forall t \geq 0
$$

\section{A.2 Talbot lemma}

Let us consider $P(x)=x^{2}-\left(a_{1}+b_{1} i\right) x+\left(a_{0}+b_{0} i\right)$. We apply Talbot Algorithm ([39]) on $P(x)$.

Let us first observe that the equation $P(x)=0$ has a purely imaginary root if and only if

$$
b_{1}^{2}+4 a_{0} \geq 0 \text { and }\left\{\begin{array}{l}
a_{1}=0 \text { and } b_{0}=0 \\
\text { or } \\
a_{1} \neq 0 \text { and } b_{0}^{2}-a_{1} b_{1} b_{0}-a_{0} a_{1}^{2}=0 .
\end{array}\right.
$$

Next let us consider

$$
f(x)=i^{2} P(-i x)=-P(-i x)=f_{0}(x)+i f_{1}(x),
$$

where $f_{0}$ and $f_{1}$ are given by

$$
\begin{aligned}
& f_{0}(x)=x^{2}+x b_{1}-a_{0}, \\
& f_{1}(x)=-a_{1} x-b_{0} .
\end{aligned}
$$

Next Euclide factorization enables to write down

$$
\begin{aligned}
& f_{0}(x)=f_{1}(x) q_{1}(x)-f_{2}(x), \\
& f_{1}(x)=q_{2}(x) f_{2}(x),
\end{aligned}
$$

where if $a_{1}\left(a_{0}-\frac{b_{0}}{a_{1}}\left(\frac{b_{0}}{a_{1}}-b_{1}\right)\right) \neq 0$ we have set

$$
\begin{aligned}
& q_{1}(x)=-\frac{1}{a_{1}} x+\frac{\frac{b_{0}}{a_{1}}-b_{1}}{a_{1}} \text { and } q_{2}(x)=\frac{a_{1} x+b_{0}}{a_{0}+\frac{b_{0} b_{1}}{a_{1}}-\frac{b_{0}^{2}}{a_{1}^{2}}} \\
& f_{2}(x)=a_{0}-\frac{b_{0}}{a_{1}}\left(\frac{b_{0}}{a_{1}}-b_{1}\right) .
\end{aligned}
$$

Let us define $p_{k}$ as $p_{k}=d^{\circ}\left(f_{k-1}\right)-d^{\circ}\left(f_{k}\right)$ and $c_{k}=\operatorname{sign}\left(\frac{f_{k-1}^{0}(x)}{f_{k}^{0}(x)}\right)$, where $f_{k}^{0}$ is the leading coefficient of $f_{k}$.

Talbot's table can be written, in the case $a_{1}\left(a_{0}-\frac{b_{0}}{a_{1}}\left(\frac{b_{0}}{a_{1}}-b_{1}\right)\right) \neq 0$, as follows:

\begin{tabular}{|c|c|c|c|}
\hline$k$ & $f_{k}(x)$ & $p_{k}$ & $\operatorname{sign}\left(c_{k}\right)$ \\
\hline 0 & $1-b_{1} a_{0}$ & & $\operatorname{sign}\left(-\frac{1}{a_{1}}\right)$ \\
1 & $-a_{1} b_{0}$ & 1 & $\operatorname{sign}\left(\frac{-a_{1}}{a_{0}-\frac{b_{0}}{a_{1}}\left(\frac{b_{0}}{a_{1}}-b_{1}\right)}\right)$ \\
\hline & $a_{0}-\frac{b_{0}}{a_{1}}\left(\frac{b_{0}}{a_{1}}-b_{1}\right)$ & 1 & \\
\hline
\end{tabular}


The number of right-half plane zeros is given by

$$
r(P)= \begin{cases}1-\frac{1}{2}\left[\operatorname{sign}\left(-\frac{1}{a_{1}}\right)+\operatorname{sign}\left(\frac{-a_{1}}{a_{0}-\frac{b_{0}}{a_{1}}\left(\frac{b_{0}}{a_{1}}-b_{1}\right)}\right)\right], & \text { if } a_{1}\left(a_{0}-\frac{b_{0}}{a_{1}}\left(\frac{b_{0}}{a_{1}}-b_{1}\right)\right) \neq 0 \\ 1+\frac{1+\operatorname{sign}(a 1)}{2}, & \text { if } a_{1} \neq 0 \text { and }\left(a_{0}-\frac{b_{0}}{a_{1}}\left(\frac{b_{0}}{a_{1}}-b_{1}\right)\right)=0 . \\ 1, & \text { if } a_{1}=0\end{cases}
$$

\section{REFERENCES}

[1] H. d'Albis, E. Augeraud-Véron and H.J. Hupkes, Discontinuous initial value problems for functional differential-algebraic equations of mixed-type. J. Differ. Equ. 253 (2012) 1959-2024.

[2] H. d'Albis , E. Augeraud-Véron and H.J. Hupkes, Multiple solutions in systems of functional differential equations. J. Math. Econ. 52 (2014) 50-56.

[3] W. Arendt and A. Favini, Integrated solutions to implicit differential equations, partial differential equations. I (Turin, 1993). Rend. Sem. Mat. Univ. Politec. Torino 51 (1993) 315-329.

[4] J. Benhabib and R.E.A. Farmer, Indeterminacy and increasing returns. J. Econ. Theory 63 (1994) 19-41.

[5] R. Bennett and R.E.A. Farmer, Indeterminacy with non-separable utility. J. Econ. Theory 93 (2000) $118-143$.

[6] O.J. Blanchard and C.M. Kahn, The solution of linear difference model under rational expectations. Econometrica 48 (1980) $1305-1312$.

[7] S. Bosi and T. Seegmuller, Can heterogeneous preferences stabilize endogenous fluctuations? J. Econ. Dyn. Control 32 (2008) 624-647.

[8] R. Boucekkine, C. Carmacho and B. Zou, Bridging the gap between growth theory and economic geography: the spatial Ramsey model. Macroecon. Dyn. 13 (2009) 20-45.

[9] R. Boucekkine, C. Carmacho and G. Fabbri, Spatial dynamics and convergence: the spatial AK model. J. Econ. Theory 148 (2013) 2719-2736.

[10] P. Brito, A spatial Solow model with unbounded growth. Working Papers, Department of Economics, ISEG (2004).

[11] P. Brito, The dynamics of distribution in a spatially heterogeneous world. Working Papers, Department of Economics, ISEG (2004).

[12] W.A. Brock and A. Xepapadeas, Pattern formation, spatial externalities and regulation in coupled economic-ecological systems. J. Environ. Econ. Manag. 59 (2008) 149-164.

[13] W.A. Brock and A. Xepapadeas, General pattern formation in recursive dynamical systems models in economics. Fondazione Eni Enrio Mattei Working Paper 49 (2009).

[14] W.H. Buiter, Saddlepoint problems in continuous time rational expectations models: a general method and some macroeconomic examples. Econometrica 52 (1984) 665-680.

[15] C. Camacho and B. Zou, The spatial Solow model. Econ. Bull. 18 (2004) 1-11.

[16] L. Chincarini and N. Asherie, An analytical model for the formation of economic clusters. Regl. Sci. Urban Econ. 38 (2008) $252-270$.

[17] W.A. Coppel, Dichotomies in stability theory. Lecture Notes in Math. Springer-Verlag, Berlin (1978).

[18] K. Desmet and E. Rossi-Hansberg, Spatial growth and industry age. J. Econ. Theory 144 (2009) $2477-2502$.

[19] K. Desmet and E. Rossi-Hansberg, On spatial dynamics. J. Regl. Sci. 50 (2010) 43-63.

[20] A. Ducrot, P. Magal and O. Seydi, Persistence of exponential trichotomy for linear operators: a Lyapunov-Perron approach. J. Dyn. Differ. Equ. 28 (2016) 93-126.

[21] W. Easterly and R. Levine, It's not factor accumulation: stylized facts and growth models. World Bank Econ. Rev. 15 (2001) $177-219$.

[22] C. Ertur and W. Koch, Growth, technological interdependence and spatial externalities: theory and evidence. J. Appl. Econometr. 22 (2007) 1033-1062.

[23] G. Fabbri, Geographical structure and convergence: a note on geometry in spatial growth models. J. Econ. Theory 162 (2016) $114-136$.

[24] M. Fugita, P. Krugman and A. Venables, The Spatial Economy. The MIT Press, Cambridge, MA (1999).

[25] X. Gabais, Zipf's law for cities: an explanation. Quar. J. Econ. 114 (1999) 739-767.

[26] X. Gabais, Zipf's law and the growth of cities. Am. Econ. Rev. 89 (1999) 129-132.

[27] C. Ghiglino and M. Olszak-Duquenne, On the impact of heterogeneity on indeterminacy. Int. Econ. Rev. 46 (2005) 171-188.

[28] B. Herrendorf, R. Waldemann and A. Valentiny, Ruling out multiplicity and indeterminacy: the role of heterogeneity. Rev. Econ. Stud. 67 (2000) 295-307.

[29] H.J. Hupkes and E. Augeraud-Véron, Well-posedness of initial value problems for functional differential and algebraic equations of mixed type. Disc. Contin. Dyn. Syst. A 30 (2011) 737-765.

[30] W. Keller, Geographic localization of international technology diffusion. Am. Econ. Rev. 92 (2002) 120-142. 
[31] P.J. Klenow and A. Rodriguez-Clare, Externalities and growth, in Handbook of Economic Growth, edited by P. Aghion, S. Durlauf. Elsevier, Amsterdam (2005) 817-861.

[32] P. Krugman, The Self-Organizing Economy. Blackwell Publishers, Cambridge, MA (1996).

[33] L.H. Loomis and S. Sternberg, Advanced calculus. Addison-Wesley, Reading, Massachusetts (1968).

[34] R.E. Lucas Jr., Externalities and cities. Rev. Econ. Dyn. 4 (2001) 245-274.

[35] H.M. Polemarchakis, Portfolio choices, exchange rates, and indeterminacy. J. Econ. Theory 46 (1988) $414-421$.

[36] D.T. Quah, Regional convergence clusters across Europe. Eur. Econ. Rev. 40 (1996) 951-958.

[37] D.T. Quah, Spatial agglomeration dynamics. AEA Papers Proc. 92 (2002) 247-252.

[38] S.J. Rey and B.D. Montouri, U.S. regional income convergence: a spatial econometric perspective. Regl. Stud. 33 (1999) $145-156$.

[39] A. Talbot, The number of zeros of a polynomial in a half-plane. Proc. Camb. Philos. Soc. 56 (1960) $132-147$.

[40] M.C. Zhou and Y.F. Yang, An application of traveling wave analysis in economic growth model. Appl. Math. Comput. 200 (2008) 261-266. 